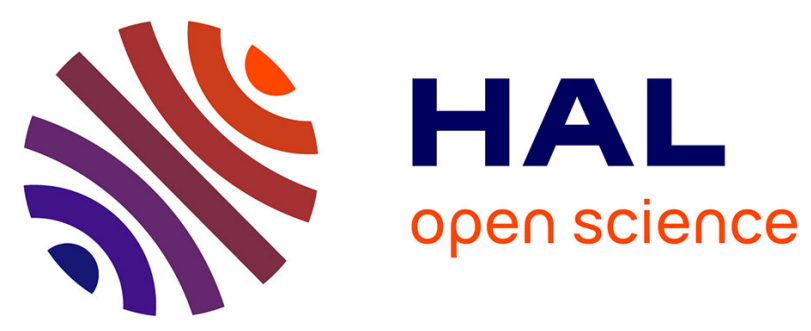

\title{
Complex coacervation of natural sophorolipid bolaamphiphile micelles with cationic polyelectrolytes
}

Ghazi Ben Messaoud, Lyndsay Promeneur, Martha Brennich, Sophie L. K. W. Roelants, Patrick Le Griel, Niki Baccile

\section{- To cite this version:}

Ghazi Ben Messaoud, Lyndsay Promeneur, Martha Brennich, Sophie L. K. W. Roelants, Patrick Le Griel, et al.. Complex coacervation of natural sophorolipid bolaamphiphile micelles with cationic polyelectrolytes. Green Chemistry, In press, 20, pp.3371-3385 10.1039/C8GC01531G . hal-01829572

\section{HAL Id: hal-01829572 \\ https://hal.sorbonne-universite.fr/hal-01829572}

Submitted on 4 Jul 2018

HAL is a multi-disciplinary open access archive for the deposit and dissemination of scientific research documents, whether they are published or not. The documents may come from teaching and research institutions in France or abroad, or from public or private research centers.
L'archive ouverte pluridisciplinaire HAL, est destinée au dépôt et à la diffusion de documents scientifiques de niveau recherche, publiés ou non, émanant des établissements d'enseignement et de recherche français ou étrangers, des laboratoires publics ou privés. 


\section{Complex coacervation of natural sophorolipid bolaamphiphile}

2 micelles with cationic polyelectrolytes

3 Ghazi Ben Messaoud, ${ }^{\mathrm{a}}$ Lyndsay Promeneur, ${ }^{\mathrm{a}}$ Martha Brennich, ${ }^{\mathrm{b}}$ Sophie Roelants,,${ }^{\mathrm{c} d}$ Patrick

$4 \quad$ Le Griel, ${ }^{a}$ Niki Baccile ${ }^{\mathrm{a}, *}$

5

6 a Sorbonne Université, Centre National de la Recherche Scientifique, Laboratoire de Chimie

7 de la Matière Condensée de Paris, LCMCP, F-75005 Paris, France

$8{ }^{\mathrm{b}}$ European Molecular Biology Laboratory, Synchrotron Crystallography Group, 71 Avenue 9 des Martyrs, 38042 Grenoble, France.

$10{ }^{\mathrm{c}}$ Ghent University, Centre for Industrial Biotechnology and Biocatalysis (InBio.be), Coupure

11 Links 653, Ghent, Oost-Vlaanderen, BE 9000

12 d Bio Base Europe Pilot Plant, Rodenhuizekaai 1, Ghent, Oost-Vlaanderen, BE 9000

$15 *$ Corresponding author:

16 Dr. Niki Baccile

17 E-mail address: niki.baccile@upmc.fr 


\section{Abstract}

Complex coacervation of polyelectrolyte with surfactant micelles is a promising system for a wide range of applications. However, the development of "green coacervates", from biobased surfactant and biopolymers has not been explored, yet. Herein, complex coacervation of natural micelles from a bolaform sophorolipid biosurfactant with oppositely charged cationic polyelectrolytes (i.e., chitosan oligosaccharide lactate, poly (L-lysine) and poly(allylamine)) was investigated. Turbidity titration, light and scanning electron microscopy (SEM), dynamic light scattering (DLS), cryogenic transmission electron microscopy (cryo-TEM) and Small Angle X-ray Scattering (SAXS) were used to monitor the evolution of complex structures as function of $\mathrm{pH}$ and polyelectrolyte concentration. Phase boundaries of the biosurfactantpolyelectrolyte systems were obtained and revealed the feasibility of coacervation in water over a broad $\mathrm{pH}$ range, from $\mathrm{pH} 5$ to $\mathrm{pH}$ 9. The state of complexation was found to depend primarily on $\mathrm{pH}$ and concentration and the used polyelectrolyte. Light microscopy and SEM demonstrated the associative macrophase separation and cryo-TEM highlighted the influence of the desolvation level on the coacervates arrangement where two main structures were formed as function of the coacervation stage namely spherical particles and aggregates. The SAXS data demonstrated that the sophorolipid micelles maintained their structure integrity following their binding to the cationic polyelectrolyte.

Keywords: Bolaform; Sophorolipid; Complex coacervation; Polyelectrolyte, Cryo-TEM.

\section{Introduction}

Glycolipids, composed of a carbohydrate-based hydrophilic head covalently linked to a fatty acid or a fatty alcohol, ${ }^{1}$ are an interesting alternative to conventional surfactants because of their biobased and renewable origin, good biocompatibility and biodegradability. ${ }^{2-4}$ Among them, microbial glycolipids like sophorolipids are an attractive class of molecules which are obtained from exclusively renewable agro-resources (rapeseed oil, oleic acid, carbohydrates) through a fermentation process of the yeast Starmerella bombicola with remarkable production rates (upper to 300 g. $\left.\mathrm{L}^{-1}\right)^{5}$ and reduced environmental impact biosynthesis. ${ }^{6,7}$ These molecules possess several potential applications ${ }^{8}$ in $\operatorname{cosmetic}^{9}$ and anticancer, ${ }^{10}$ but also like a structuring agent for self-assembled nanomaterials, ${ }^{11}$ or as antimicrobial agents. ${ }^{12}$ Nevertheless, the development of novel applications involving sophorolipids in particular, and microbial glycolipids in general, may undergo through the investigation of their binding to further colloidal species or macromolecules, as shown by Dubey et $a l$., who reported that the gelation kinetic of silk fibroin can be triggered by sophorolipids ${ }^{13}$ or by Madsen et al., who 
demonstrated that the thermal stability of a lipase from Thermomyces lanuginosus can be enhanced following binding to rhamnolipids and sophorolipids. ${ }^{14}$ In this context, the phase behavior of polymer-surfactant mixtures is of a great importance for both scientific and industrial fields since they are frequently used in various formulations for foods, detergents and cosmetics. ${ }^{15}$ Their molecular association could lead to the formation of a wide range of structures like gels, micelle-decorated network, aggregates, complexes and precipitates. ${ }^{15,16}$ By controlling the interaction between surfactants and polymers, it is also possible to induce complex coacervation, a process during which a homogeneous macromolecular aqueous solution undergoes an associative liquid-liquid phase separation. ${ }^{17}$

Coacervation is considered as an eco-friendly process as it usually takes place in water and at relatively mild conditions of $\mathrm{pH}$ and temperature. In addition, it is also a cost-effective technique since neither a special device nor extensive production steps are required. The obtained structures induced by demixing are considered among the more intriguing systems in colloid chemistry. ${ }^{18-20}$ Their exotic character attracted scientists even beyond the field of colloidal chemistry like Oparin, a Russian biologist, who proposed that coacervates could be the origin of life on earth ${ }^{21}$ and some recent studies are heading towards the same direction. ${ }^{22-}$ 24,25,26 This process was initially reported by Tiebackx in 1911 without using the word, ${ }^{27}$ he found that the addition of an acid to a mixed solution of Arabic gum and gelatin results in phase separation. It was almost two decades later that the term "coacervation" was coined by Bungenberg de Jong and Kruyt who studied the phase behavior of several binary mixtures by optical microscopy. ${ }^{17}$

Typically, this phenomenon can be divided into "simple" and "complex". Simple coacervation involves only one colloidal specie or macromolecule and can be achieved through self-charge neutralization by the addition of dehydrating agents like alcohols ${ }^{28}$ and salts. ${ }^{29}$ Complex coacervation, on the contrary, consists of more than one macromolecular component $^{30,31}$ and it can occur between polyelectrolytes and oppositely charged polyelectroytes, ${ }^{32-37}$ proteins $^{38-40}$ dendrimers $^{41}$ or micelles. ${ }^{42-47}$ The preparation of coacervates has gained a lot of interest due to their broad range of applications in food, ${ }^{39}$ tissue engineering, ${ }^{48,49}$ drug delivery, ${ }^{50}$ underwater adhesives, ${ }^{51,52}$ porous material ${ }^{53}$ and water treatment. $^{54,55}$

Commonly, surfactant-polymer coacervation can occur between nonionic surfactant and nonionic polymer or polyelectrolyte but also between ionic surfactant and nonionic polymers, or polyelectrolyte. ${ }^{56}$ However complex coacervation between oppositely charged polyelectrolytes and surfactants has drawn much more attention due to the broad applications 
in technological areas. ${ }^{57}$ To date, the surfactant types used to induce complex coacervation are usually single chain or gemini type which are composed of a long hydrocarbon chain, an ionic group, a rigid spacer, a second ionic group and another hydrocarbon tail. ${ }^{56}$

In the context of complex coacervation between oppositely charged surfactant and polymers, the electrostatic binding must be strong enough to induce coacervation, but not too strong, otherwise precipitation occurs. ${ }^{56}$ To overcome potential precipitation by excessive electrostatic interaction, Dubin and co-workers, developed an interesting strategy based on the preparation of mixed micelles from anionic (sodium dodecylsulfate), or cationic (dodecyltrimethylammonium bromide), surfactant with a nonionic surfactant (Triton X-100). By adjusting the mole fraction of the charged surfactant, they were able to induce and to control the coacervation process with cationic (poly-(dimethyldiallylammonium chloride) or anionic (poly(sodium styrenesulfonate) or poly(sodium acrylate)) polyelectrolytes. ${ }^{47,58,59}$

This process depends generally on many physicochemical parameters like temperature, $\mathrm{pH}$, charge ratio of the macroions and colloid properties like molecular weight and chain flexibility ${ }^{60,61}$ However, it was found that a minimum salt addition is required to modulate the interaction strength between micelles and cationic polyelectrolyte (PEC) by charge screening, or to enhance the PEC chain flexibility by decreasing intra-chain electrostatic repulsion. ${ }^{42}$

Considering the increasing restrictions in terms of using chemical surfactants, the development of coacervates based on biobased amphiphiles and biomacromolecules becomes an important challenge. Therein, Imura et al., found that mannosylerythritol lipids, a glycolipid biosurfactant, can form spontaneous coacervates in water. ${ }^{62}$ These structures are obtained by simple coacervation and are induced by efficient dehydration in water. Nonetheless, the complex coacervation, a phenomenon with great interest in industry, between macromolecules and glycolipids has not been reported in this context, yet.

Herein, we develop green complex coacervates based on a microbial biosurfactant and a set of three polymers, including one bio-derived polymer. We use a bolaform acidic sophorolipid (SL) constituted of a sophorose (glucose $\beta(1,2)$ ) linked to the $\mathrm{C} 17$ atom of oleic acid via an acetal bond (Figure 1a). The study was also motivated by the original micellar structure reported for sophorolipids and to the fact that the carboxylic group is being free of access at the opposite side of the molecule (Figure 1b). ${ }^{63}$ Unlike conventional surfactants, where the coacervation process is controlled by the surfactant molar fraction, salt or by the addition of a nonionic surfactant using Dubin's strategy, SL has itself a tunable charge, for its $\mathrm{pH}$ sensitivity ( $\mathrm{pKa}=5.8$ ); therefore, the global charge of the resulting micelles could be easily handled just by $\mathrm{pH}$ without the need of a second further surfactant, or other additives. ${ }^{63}$ 

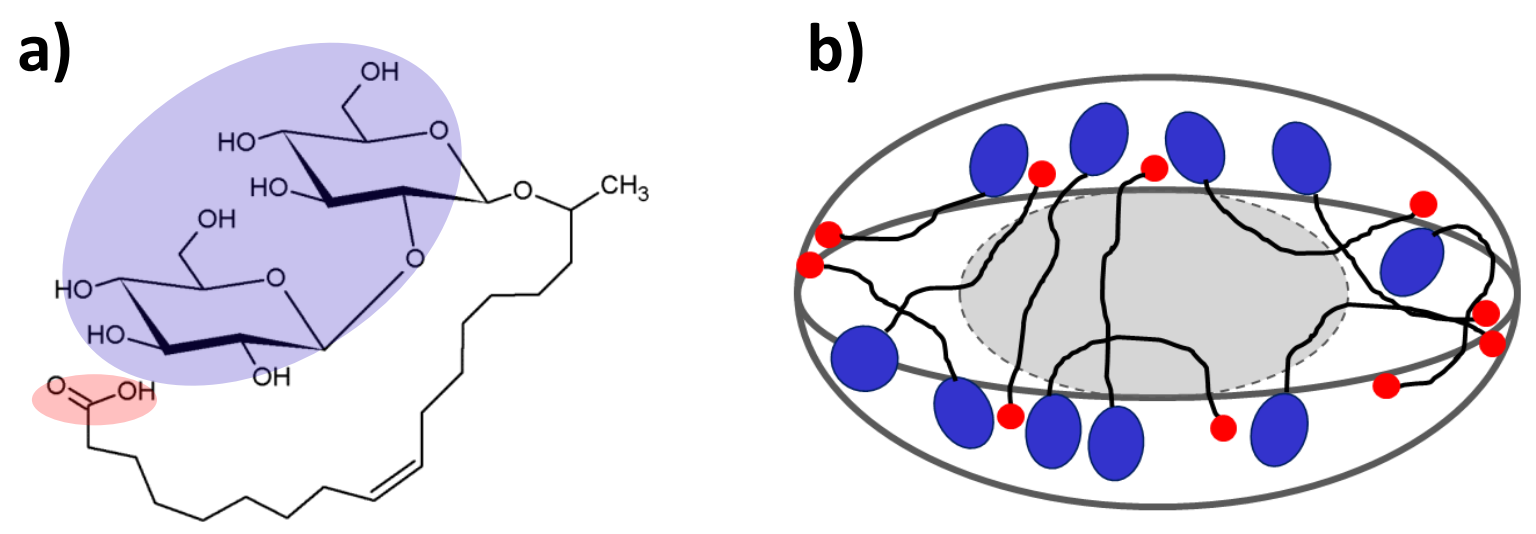

c)

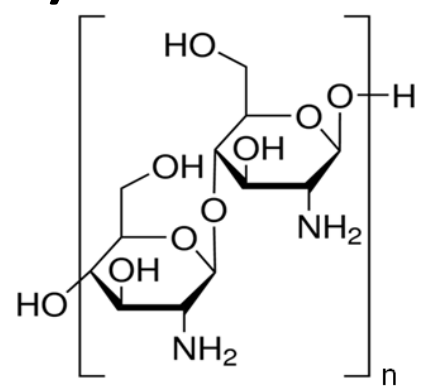

d)

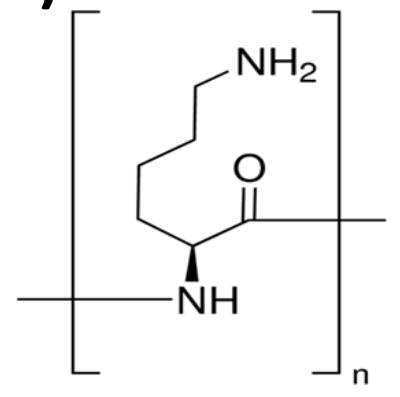

e)

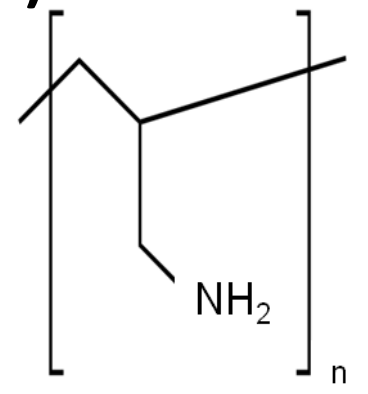

Figure 1 - (a) Chemical structure of acidic form of SL; (b) model of the structure of the micelle formed from SL alone at equilibrium in water (red dot and blue ellipse respectively schematize the COOH and sophorose groups in the SL molecule in a)) and chemical structure of (c) CHL, (d) PLL and (e) PAA polyamines.

In the field of complex coacervation, no studies between polymers and bolaamphiphile micelles have been reported yet. In the meanwhile, complex coacervation between polymers and surfactants has never been proposed in the context of green chemistry, where both the amphiphile and the polymer are biosourced. Here, the behavior of SL micelles was studied in the presence of three water-soluble polyamines, ${ }^{35-37}$ chitosan oligosaccharide lactate (CHL), poly(L-lysine) (PLL) and poly(allylamine) (PAA) (Figure 1c-e): CHL is a chitosan derivative, a cationic polysaccharide obtained by deacetylation of chitin which is a structural polysaccharide of insects and crustaceans shells. Chitosan is well-known for its wide availability, biocompatibility, biodegradability and poor toxicity. ${ }^{64}$ PLL and PAA are synthetic polymers, although the former is based on peptide coupling of the amino acid lysine, while the latter is a classical petrochemical polymer based on allylamine. ${ }^{65}$ These polymers have been chosen on the following basis: CHL is a semi-flexible biopolymer, while PLL and 
PAA are flexible synthetic polymers. ${ }^{65}$ The comparison between the three polyelectrolytes, although of different chemical origin, shows the broad validity of the complex coacervation using SL micelles.

In the current paper, we explore the complex coacervation of SL micelles with the PEC mentioned above. The influence of $\mathrm{pH}$, as well as PEC concentration was monitored by turbidimetric titration and dynamic light scattering (DLS). The resulting structures were examined by small angle X-ray scattering (SAXS), cryo-transmission electron microscopy (cryo-TEM), scanning electron microscopy (SEM) and optical microscopy.

\section{Experimental Section}

\section{Chemicals}

SL were purchased from Soliance (Givaudan Active Beauty, France) and hydrolyzed in alkaline medium and the $\mathrm{pH}$ was then decreased to around 4.5 to obtain the open acidic form and finally recovered using method 1 as reported previously. ${ }^{66}$ The purity is evaluated at about $90 \%$ of both terminal and sub-terminal C18:1 congeners and their equilibrium state in water is micellar, as described elsewhere. ${ }^{67,68,69}$ The critical micellization concentration $(\mathrm{cmc})$ is around $0.1 \mathrm{mg} / \mathrm{mL}$ at room temperature. CHL $\left(\mathrm{M}_{n} \approx 5 \mathrm{kDa}, \mathrm{pKa} \approx 6.5\right)^{70}$ with a deacetylation degree $>90 \%$, PLL hydrobromide $\left(\mathrm{M}_{w} \approx 1-5 \mathrm{kDa}, \mathrm{pKa} \approx 10\right)^{71}$ and PAA hydrochloride $\left(\mathrm{M}_{w} \approx 17.5 \mathrm{kDa}, \mathrm{pKa} \approx 9.5\right)^{71}$ were purchased from Sigma-Aldrich. All other chemicals were of reagent grade and were used without further purification.

\section{Preparation of solutions}

SL $(10$ or $50 \mathrm{mg} / \mathrm{mL})$, CHL (4 mg/mL), PLL $(10 \mathrm{mg} / \mathrm{mL})$ and PAA $(4 \mathrm{mg} / \mathrm{mL})$ stock solutions were prepared by dispersing the former in Milli-Q-grade water. The solutions were stirred until complete hydration. The $\mathrm{pH}$ of $\mathrm{SL}(10 \mathrm{mg} / \mathrm{mL})$ and the cationic PEC solutions was typically between 3 and 4 except for PLL solution $(\mathrm{pH}>5.5)$, for which it was decreased to 3.5 by adding $2 \mu \mathrm{L}$ of $\mathrm{HCl}(1 \mathrm{M})$.

\section{Turbidimetric titration}

The influence of $\mathrm{pH}$ and cationic molecules concentration on the formation of coacervates droplets was investigated by measuring the absorbance at a wavelength range of 400-700 nm. The turbidity was then reported as $100-\% \mathrm{~T}$ (where $\mathrm{T}$ is the transmittance and is equal to $10^{-\mathrm{A}}$ and $\mathrm{A}$ is the absorbance at $450 \mathrm{~nm}$ ). Data were recorded at room temperature using a UV/Vis spectrophotometer (UVIKON XL, BioTeK) and a UV-Vis-NIR spectrophotometer (Cary 
5000, Agilent Technologies) for some experiments.

To study the influence of $\mathrm{pH}$, equal volumes of SL and PEC solutions were mixed after appropriate dilution of the stock solutions with varying final concentrations range for CHL (0.5-2 $\mathrm{mg} / \mathrm{mL})$, PLL $(1-5 \mathrm{mg} / \mathrm{mL})$ and PAA $(0.3-2 \mathrm{mg} / \mathrm{mL})$ while the final concentration of SL solution was kept constant $(5 \mathrm{mg} / \mathrm{mL})$. Next, the $\mathrm{pH}$ of $2 \mathrm{~mL}$ of each mixture was increased progressively by the addition of small amounts $(2,5$ or $10 \mu \mathrm{L})$ of $\mathrm{NaOH} 0.1 \mathrm{M}$ under gentle stirring and the final turbidity curves were recorded only after complete homogenization of the solution and $\mathrm{pH}$ stabilization. It is worth mentioning that the turbidity of pure compound solutions was also measured as function of $\mathrm{pH}$.

The coacervation dependence on SL concentration was measured at optimal $\mathrm{pH}-$ values for CHL (pH 5.6, $1.4 \mathrm{mg} / \mathrm{mL}$ ), PLL (pH 6.2, 2 mg/mL) and PAA (pH 6.3, $0.75 \mathrm{mg} / \mathrm{mL}$ ) by stepwise additions ( 2 or $5 \mu \mathrm{L}$ ) of SL solution $(50 \mathrm{mg} / \mathrm{mL})$ with the corresponding $\mathrm{pH}$ under gentle stirring. The final turbidity curves were recorded only after complete homogenization of the mixture and the $\mathrm{pH}$ was regularly measured and adjusted if necessary with $\mathrm{NaOH}(1 \mathrm{M})$.

\section{Dynamic Light Scattering measurements (DLS)}

Size distribution and electrophoretic mobility of SL free micelles and SL-PEC complexes as a function of $\mathrm{pH}$ were measured by DLS using a Malvern Zetasizer Nano ZS90 (Malvern Instruments Ltd, Worcestershire, UK) equipped with a $4 \mathrm{~mW}$ He-Ne laser at a wavelength of $633 \mathrm{~nm}$. Measurements were made at $25^{\circ} \mathrm{C}$ with a fixed angle of $90^{\circ}$ and three acquisitions of 15 measurements per sample. Although zeta potential $(\zeta)$ calculated using Smoluchowski, Hückel, or Henry equations is a more common way to quantify surface charge, no attempt has been made to convert the electrophoretic mobility values $(\mu)$ into $\zeta$ because the complex system composed of SL micelles, free polymer and SL-PEC coacervates cannot be described by the usual, simple, hard-sphere model, hypothesized in standard theories relating $\mu$ to $\zeta{ }^{72}$

\section{Small Angle X-ray Scattering (SAXS)}

SAXS experiments are performed at $25^{\circ} \mathrm{C}$ immediately after sample preparation on the BioSAXS BM29 beamline at the ESRF synchrotron facility (Grenoble, France) using 12.5 $\mathrm{KeV}$ energy and a sample-to-detector distance of $2.867 \mathrm{~m}$, the beamline standard configuration. The energy is calibrated by measuring the $\mathrm{L}_{\mathrm{I}}$ and $\mathrm{L}_{\mathrm{III}}$ edges of platinum and the sample-to-detector distance is determined using silver behenate $\left(d_{\text {ref }}=58.38 \AA\right)$. (http://www.esrf.eu/home/UsersAndScience/Experiments/MX/About_our_beamlines/bm29.ht 
ml). ${ }^{73}$ For this experiment, we employ the automatic sample changer for liquids using the 96well plates and about $100 \mu \mathrm{L}$ of each sample. ${ }^{74}$ The liquid sample is automatically loaded into a $1.8 \mathrm{~mm}$ quartz glass capillary and ten acquisitions of $1 \mathrm{~s}$ each are taken as the sample passes the beam. Individual frames are manually controlled for systematic changes and averaged for better statistics if none are found. Eventual changes can be either due to intrinsic sample heterogeneity or radiation damage. The signal of the Pilatus 1M 2D detector, used to record the data, is integrated azimuthally with PyFAI to obtain the $I(q)$ vs. $q$ spectrum $(q=$ $4 \pi \sin \theta / \lambda$, where $2 \theta$ is the scattering angle) after masking systematically wrong pixels and the beam stop shadow. ${ }^{75}$ Absolute intensity units were determined by measuring the scattering signal of water $\left(0.0163 \mathrm{~cm}^{-1}\right)$.

\section{Coacervates imaging}

Light Microscopy. To highlight the coacervate droplets, images were acquired using a Nikon DS-Ri1 optical microscope in Brightfield mode and a Zeiss AxioImager D1 microscope in differential interference contrast (DIC) mode.

Scanning Electron Microscopy (SEM). The coacervates solutions were freeze-dried during 48 hours and the obtained samples were observed using a Hitachi (S-3400N) electron microscope operating at $3 \mathrm{kV}$.

Cryogenic Transmission Electron Microscopy (Cryo-TEM). These experiments were carried out on an FEI Tecnai 120 twin microscope operating at $120 \mathrm{kV}$ equipped with a Gatan Orius CCD numeric camera. The sample holder was a Gatan Cryoholder (Gatan 626DH, Gatan). On both microscopes, Digital Micrograph software was used for image acquisition. Cryofixation was done on a homemade cryofixation device. The solutions were deposited on a glow-discharged holey carbon coated TEM copper grid (Quantifoil R2/2, Germany). Excess solution was removed and the grid was immediately plunged into liquid ethane at $-180{ }^{\circ} \mathrm{C}$ before transferring them into liquid nitrogen. All grids were kept at liquid nitrogen temperature throughout all experimentation.

\section{Quantification of coacervation}

Nuclear Magnetic Resonance (NMR): solution NMR has been here used to quantify both the extent of coacervation and the ratio between the $\mathrm{COOH}$ of $\mathrm{SL}$ and $\mathrm{NH}_{2}$ of the polyelectrolytes given in Figure 1. One should note that in the rest of the manuscript we will broadly refer to them as $\mathrm{C}=\mathrm{O}$ and $\mathrm{NH}_{\mathrm{x}}$, which respectively account for the carboxylic/carboxylate and 
amino/ammonium groups. Solution NMR is a technique being sensitive to molecular species with fast tumbling in solution. In this work, NMR is mainly sensitive to both SL and polyelectrolyte in solution, not associated in complex coacervates: out of the coacervation region, NMR is sensitive to the entire SL and polyelectrolyte population, while in the coacervation region, NMR is only sensitive to SL and polyelectrolyte in equilibrium with the complex coacervates. Quantification is probed with two different methods; in the first one, solutions containing the coacervates are analysed directly by ${ }^{1} \mathrm{H}$ NMR. As controls, we have analysed the SL-polyelectrolyte mixtures out of the coacervation region as well as the single components within and out of the coacervation region. In the second approach, the coacervates are centrifuged out of the solution. The supernatant is analysed as such while the coacervates are redispersed in the same volume of water at a $\mathrm{pH}$ set out of the coacervation region, so to detect the entire SL and polyelectrolyte population.

For the first set of experiments, we have studied each control solution individually (SL, CHL, PLL, PAA) and their association (SL-CHL, SL-PLL, SL-PAA). All solutions are freshly prepared in $\mathrm{D}_{2} \mathrm{O}$ at $\mathrm{pH}(\mathrm{pD})$ values below and above the known $\mathrm{pH}$ of coacervation, determined according to the turbidity data. One should note that we do not experience any difference when using deuterated instead of hydrogenated water. $1 \mathrm{M} \mathrm{NaOD}$ and $1 \mathrm{M} \mathrm{DCl}$ solutions have been employed to change pD. Controls: SL (5 mg/mL, pD: 4.36, 6.33), CHL (1.4 mg/mL, pD: 3.80, 6.09), PLL (2 mg/mL, pD: 4.58, 7.14) and PAA (0.75 mg/mL, pD: $3.91,6.23)$. For the study of the coacervates, we have used exactly the same concentration values employed for the controls and the following $\mathrm{pD}$ values: $\mathrm{SL}-\mathrm{CHL}, \mathrm{pD}=4.46,6.12$; $\mathrm{SL}-$ PAA, $\mathrm{pD}=4.00,6.00 ; \mathrm{SL} / \mathrm{PLL}, \mathrm{pD}=4.35,7.00$. In the second set of experiments, we have centrifuged the coacervate (SL-CHL, SL-PLL and SL-PAA solutions at pD>6) at $3000 \mathrm{rpm}$ for $1 \mathrm{~h}$, a condition which is known to separate efficiently the colloid-rich phase without destructuring the coacervates. ${ }^{43}$ The supernatant has been removed and analyzed as such while the coacervate has been redispersed in $500 \mu \mathrm{L} \mathrm{D}_{2} \mathrm{O}$ at $\mathrm{pH}$ below 6 , out of the coacervation region, as explained above.

All ${ }^{1} \mathrm{H}$ solution NMR experiments are acquired on a Bruker Avance III 300 spectrometer using a $5 \mathrm{~mm} \mathrm{1H}-\mathrm{X}$ BBFO probe. Number of transient is 16 with $2.3 \mathrm{~s}$ recycling delay, acquisition time of $2.72 \mathrm{~s}$ and a receiver gain of 256 . We have employed a $5 \mathrm{~mm}$ NMR tube containing exactly $500 \mu \mathrm{L}$ of solution. For quantitation purposes, these conditions have been kept constant throughout all experiments.

The $\mathrm{C}=\mathrm{O} / \mathrm{NH}_{\mathrm{x}}$ ratio has been determined by the integral ratio between the $\underline{\mathrm{C}}_{2}$ groups in $\alpha$ position for $\mathrm{SL}\left(\mathrm{R}-\underline{\mathrm{CH}}_{2}-\mathrm{C}=\mathrm{O}, \delta=2.33 \mathrm{ppm}\right)$ and the polyelectrolyte $\left(\mathrm{CHL}: \mathrm{R}-\mathrm{C} \underline{\mathrm{H}}-\mathrm{NH}_{\mathrm{x}}, \delta=\right.$ 
3.16 ppm; PAA: $\mathrm{R}-\underline{\mathrm{C}}_{2}-\mathrm{NH}_{\mathrm{x}}, \delta=3.10 \mathrm{ppm}$; PLL: $\left.\mathrm{R}-\mathrm{C}_{2}-\mathrm{NH}_{\mathrm{x}}, \delta=3.00 \mathrm{ppm}\right)$ at $\mathrm{pH}<5$. The extent of coacervation is calculated by measuring the intensity loss between the SLpolyelectrolyte mixture out of the coacervation region $(\mathrm{pD}<5$, all species detected) and in the coacervation region ( $\mathrm{pD}>5$, only free $\mathrm{SL}$ and polyelectrolyte detected) in the 3-4 ppm region. The signal loss corresponds to the amount of sample in the coacervate phase. The latter is compared with the direct measurement of the intensity of the coacervate rich-phase after centrifugation and redispersion.

\section{Results and discussion}

\section{pH-induced complex coacervation}

Complex coacervation based on charge pairing occurs when positive and negative charges in the micelles and macromolecules compensate. In $\mathrm{pH}$-responsive systems, ${ }^{32}$ the ionization rate depends on $\mathrm{pH}$ and the $\mathrm{pKa}$ of the acid, or base, in the given molecule. If the $\mathbf{M}_{w}$ of SL is known, the number and weight average molecular weight $\left(\mathbf{M}_{n}\right.$ and $\left.\mathbf{M}_{w}\right)$ of the commercial PEC used here are only estimated. For this reason, although one can estimate the relative mass fraction values of SL and PEC needed to obtain a 1:1 molar ratio of their ionizable groups $\left(\mathrm{COOH}\right.$ and $\left.\mathrm{NH}_{2}\right)$, a variation in the relative concentrations is necessary. Similarly, the differences in the pKa values among all molecular partners suggest that if complex coacervation occurs, that will be strongly $\mathrm{pH}$-dependent. For these reasons, complex coacervation was qualitatively determined using turbidity measurements as a function of $\mathrm{pH}$ and PEC concentration for a given SL amount $(5 \mathrm{mg} / \mathrm{mL}$ and $10 \mathrm{mg} / \mathrm{mL}$, respectively 8 and $16 \mathrm{mM})$.

A typical turbidimetric titration curve of SL-PAA $\left(\mathrm{C}_{\mathrm{PAA}}=0.75 \mathrm{mg} / \mathrm{mL}\right)$ is shown in Figure 2. The coacervation process as a function of $\mathrm{pH}$ can be described in terms of a set of specific $\mathrm{pH}$ values corresponding to the limits of four different regions of phase behavior. Region 1: at low pH-values, generally below $\mathrm{pH} 5$, the solution is clear and the turbidity is constant and close to zero; Region 2: an abrupt increase in turbidity from a starting $\mathrm{pH}$, designed as $\mathrm{pH} \varphi$, characterizes this region and it reflects the cloudy aspect of the solution. Region 3: this $\mathrm{pH}$ interval is characterized by a plateau from a starting $\mathrm{pH}_{\max }$ and where the turbidity is constant and maximum and where the solution shows an opalescent behavior. Region 4: the turbidity decreases progressively until a transparent solution again. As a general remark, transparency in Region 1 and Region 4 strongly depend on the solubility of each component (micelle and polymer), an aspect which will be discussed later. 


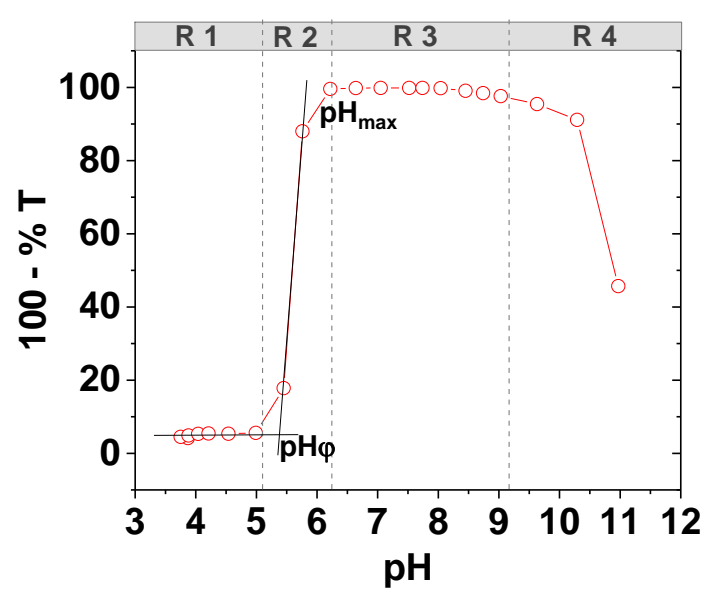

\section{Figure 2 - Turbidity $(100-\% \mathrm{~T})$ as a function of $\mathrm{pH}$ for a SL-PAA mixture. $[\mathrm{SL}]=5 \mathrm{mg} / \mathrm{mL}$; $[\mathrm{PAA}]=0.75$ $\mathrm{mg} / \mathrm{mL}$. $R$ stands for Region.}

The turbidimetric titration of SL with CHL, PLL or PAA at various PEC concentrations are presented in Figure 3a-c, while Figure S 1 and Figure S 2 show the specific system SL at $10 \mathrm{mg} / \mathrm{mL}$ and CHL in the concentration range between 0.25 and $2.0 \mathrm{mg} / \mathrm{mL}$. Figure 3 shows that any SL-PEC mixture displays the same $\mathrm{pH}$-dependent turbidity profile described in Figure 2, however the type and concentration of PEC have a strong impact on the phase transition, as discussed above, and analyzed in more detail hereafter. Figure 3a-c shows that the transition $\mathrm{pH}$ in Region 2, and the stability of Region 3 as a function of $\mathrm{pH}$, strongly depend on the SL-PEC ratio. This aspect will be discussed in more detail below. Region 4 is identified for all systems by the sudden loss in turbidity, except for SL-CHL, the turbidity of which is still high due to the insolubility of $\mathrm{CHL}$ above $\mathrm{pH}$ 7. The phenomena observed by turbidity and described above are classically-observed in oppositely charged PEC, like chitosan with Arabic gum, ${ }^{76}$ or with hyaluronic acid $^{32}$ and for PEC-protein systems, like poly(dimethyldiallylammonium chloride) with bovine serum albumin, ${ }^{77}$ Arabic gum with whey $\operatorname{protein}^{78}$ or with $\beta$-lactoglobulin. ${ }^{79}$ Micelles-PEC systems show a similar phenomenon, such as partially described for polyacrylic acid with mixed micelles of $n$-hexadecyl trimethyl ammonium chloride and $n$-dodecyl hexaoxyethylene glycol monoether ${ }^{80}$ It should be noted that $\mathrm{pH}$-induced coacervation is classically observed for oppositely charged PECs, or for PEC-protein systems, but less discussed for PEC-micelles systems. The main reason is, unlike the SL used in this work and which is $\mathrm{pH}$-sensitive $(\mathrm{pKa}=5.8)$, the charge density of most conventional surfactants, such as sodium dodecyl sulfide (SDS), are practically not sensitive to $\mathrm{pH}$ variation in a standard $\mathrm{pH}$ range range $(3<\mathrm{pH}<10)$. Else, in a number of studies implying a model system of mixed micelles (SDS-Triton X-100), authors generally use the cationic poly(diallyldimethylammoniumchloride), which is a $\mathrm{pH}$-independent polyion. ${ }^{81}$ 
One should also note that the global charge of the SL micelles is not only modulated by the deionization degree of the carboxylic groups, but also by their spatial localization, which is not clearly defined, as commonly found in standard micelles composed of ionic surfactants (e.g., SDS). A combination of scattering techniques and Molecular Dynamics (MD) simulations indicate that the carboxylic groups can be localized in a much broader and poorlydefined shell volume around the hydrophobic core. ${ }^{63}$

According to the wide literature data on complex coacervation involving oppositely charged PEC-colloids, Region 1 identifies the coexistence of two water-soluble, non- or weakly-interacting species, in this work represented by SL micelles ${ }^{63,67,68}$ and PEC. Region 2 defines the point of initial SL-PEC interaction leading to insoluble complex formation. In Region 3 the complex SL-PEC coacervate is stable at any $\mathrm{pH}$. The end of the plateau is generally related to the appearance of spurious precipitates, which could coexist with coacervates, since the turbidity remains relatively high. In Region 4, the coacervate is no longer stable. These hypotheses are confirmed by additional experiments. Figure 3d-e show the optical microscopy images recorded for SL-CHL and SL-PLL at the maximum turbidity plateau (Region 3), and they show the widespread presence of spherical droplets, which supports the idea of a liquid-liquid phase separation for all samples. Figure $3 \mathrm{f}$ and Figure $S 3$ confirm these assumptions for all systems using SEM on freeze-dried samples. Figure S 2 shows a series of crossed experiments performed on the SL-CHL system. pH-dependent electrophoretic mobility data confirm the charge-dependency of the complex coacervate formation, as the decrease in monotonic charge (at low $\mathrm{pH}$ the overall charge should be positive, as expected for the presence of positively-charged ammonium groups on PEC and neutral SL micelles) correlates with an increase in turbidity: Region 3, where charge matching should occur, is effectively characterized by the lowest charge. This behavior is typical for complex coacervates ${ }^{32}$. Macrophase separation is shown by the presence of large droplets in Figure S 2d while meso- and microscale phase separation is demonstrated by a combination of DLS and cryo-TEM, respectively indicating a colloidal dispersion having an average hydrodynamic radius of $\sim 300 \mathrm{~nm}$ (Figure S 2b) and an apparent radius in the order of $100 \mathrm{~nm}$ (Figure S 2c).

Additional tests have been done by varying the overall SL concentration. Figure S 1 shows a SL solution at $10 \mathrm{mg} / \mathrm{mL}$ for various amounts of CHL as a function of $\mathrm{pH}$. One can easily determine the presence of Regions 1 through 4 between $\mathrm{pH} 4$ and $\mathrm{pH}$ 10, whereas the lower the amount of CHL, the lower the turbidity in Region 4, above $\mathrm{pH} 7$, dependent on the insoluble CHL at neutral-basic pH. Figure S 4 goes even further as it shows the evolution of 
the turbidity as a function of the SL concentration for a constant PEC amount $\left(\mathrm{C}_{\mathrm{CHL}}=1.4\right.$ $\left.\mathrm{mg} / \mathrm{mL}, \mathrm{C}_{\mathrm{PLL}}=2 \mathrm{mg} / \mathrm{mL}, \mathrm{C}_{\mathrm{PAA}}=0.75 \mathrm{mg} / \mathrm{mL}\right)$ and $\mathrm{pH}$ values $\left(\mathrm{pH}_{\mathrm{SL}-\mathrm{CHL}}=5.6 ; \mathrm{pH}_{\mathrm{SL}-\mathrm{PLL}}=6.2\right.$; pH $\mathrm{SL}_{\mathrm{PAA}}=6.3$ ), which has been chosen to be in the stable complex coacervate phase, at the top of the plateau (Region 3) for each system, from Figure 3a-c. Figure S 4 shows that the turbidity appears after the addition of at least $1 \mathrm{mg} / \mathrm{mL}$ of SL, which is a value at which SL are already in the micellar state (cmc of SL is at least ten times lower). However, SL is added in an environment within positive charges, localized on the polymers. In this situation, the interaction between SL and PEC could reflect the following three-step mechanism, generally found in the literature: ${ }^{15,57,82}$ (a) at low surfactant concentration, the SL monomers starts to interact with the PEC chain by electrostatic binding between the carboxylic groups and cationic sites of SL and PEC, respectively (region where the turbidity remains minimal). (b) When the SL critical aggregation concentration (cac) is reached, the SL monomers will be integrated into the micelles (region where the turbidity starts to increase). (c) With further increasing of SL molecules, the binding sites of PEC are gradually saturated which will lead to complex coacervation (region of maximum turbidity). As shown in Figure $\mathrm{S} 4$, the turbidity increases abruptly for SL-PLL and SL-PAA compared to SL-CHL, for which the turbidity increases gradually. This could be explained by the differences in the relative ionization degrees of the each PEC and SL at the $\mathrm{pH}$ under study. At $\mathrm{pH}$ 6.2, the ionization degree (please refer to the Electronic Supplementary Information for more details) of PLL and PAA $(\beta)$ is unitary $(\beta=1$, as their $\mathrm{pKa} \sim 10)$; the ionization degree of SL is also high $(\alpha \sim 0.7)$. Under these conditions, each SL molecule added to the PEC solution contributes to screen the PEC charge. On the contrary, at $\mathrm{pH} 5.6$, the ionization degree of $\mathrm{CHL}$ is $\sim 0.9$, while the ionization degree of SL is lower ( $\alpha \sim 0.4)$. Under these circumstances, the charge of CHL is compensated by two SL molecules.

Even if the gradual addition of SL surfactant molecules to the PEC solution is may not reflect the same scenario if compared to that of mixing preformed SL micelles upon adding a PEC solution before $\mathrm{pH}$ titration, one can identify the SL concentration of $5 \mathrm{mg} / \mathrm{mL}(0.0080$ M) as being a good compromise for the formation of a robust SL-PEC complex coacervate in all systems. Considering the average molecular masses of each PEC used in this experiment (refer to the experimental section), one can estimate the optimal charge ratio $\left[\mathrm{COO}^{-}\right]:\left[\mathrm{NH}_{3}{ }^{+}\right]$ at which complex coacervation starts to occurs to be $0.32,0.27$ and 0.5 for, respectively, SLCHL, SL-PLL and SL-PAA systems. The detailed approach of the charge $\left[\mathrm{COO}^{-}\right]:\left[\mathrm{NH}_{3}{ }^{+}\right]$ ratio determination is described in the Electronic Supplementary Information. Even if the effective $[\mathrm{COO}]:\left[\mathrm{NH}_{3}{ }^{+}\right]$ratio is not unitary, our data are in line with literature, where non- 
stoichiometric complex coacervation can easily be found, for instance in a chitosan and hyaluronic acid mixture in a $50 \mathrm{mM} \mathrm{NaCl}$ solution and where the $\left[\mathrm{COO}^{-}\right]:\left[\mathrm{NH}_{3}{ }^{+}\right]$charge ratio varies between 0.08 and $0.72 .^{32}$ The authors attributed this observation to the chain semiflexibility and potential charge mismatch between the oppositely charge polyelctrolytes. ${ }^{32}$ Non-stoichiometric charge ratio values were also reported for PEC-micelles ${ }^{59}$ and it is generally explained as a mismatch between a calculated "macro-scale stoichiometry" and a "micro-scale stoichiometry", which is related to the effective ratio within the coacervates. Moreover, from a thermodynamic point of view, the most common way to describe complex coacervation is based on the Flory-Huggins theory which considers the change of the Gibbs free energy of mixing $\left(\Delta G_{m i x}=\Delta H_{m i x}-T \Delta S_{m i x}\right)$, where $\Delta H_{m i x}$ and $\Delta S_{m i x}$ are, respectively, the variation of enthalpy and entropy and $\mathrm{T}$ is the temperature of the mixture. ${ }^{83,84}$ The coacervation between polymers and micelles is thus driven by the enthalpy of complexation and by entropy increase, due to the release of condensed counterions; in fact, the entropic contribution is even thought to drive complex coacervation, as described by Rigsbee and Dubin. ${ }^{85}$ Using isothermal titration calorimetry, they demonstrated that both complexation and coacervation of poly-(dimethyldiallylammonium chloride) with dodecyltrimethylammonium bromide/ TritonX-100 micelles were mainly entropy-driven. Since no salt was added (generally $\mathrm{NaCl}$ is classically used), the nature and amount of counterions arising from the used PEC could impact significantly their condensation and release which will increase the entropy $\left(\Delta S_{m i x}\right)$ and affect the coacervation process. Even if it is hard to estimate the entropic contribution to complex coacervation in the SL-PEC systems studied here, one should not forget that any $\mathrm{pH}$ change involves the presence of counterions, which could play an important role in determining the exact coacervation boundary. Further experiments should be performed to confirm this hypothesis, although they are not the scope of this work. 

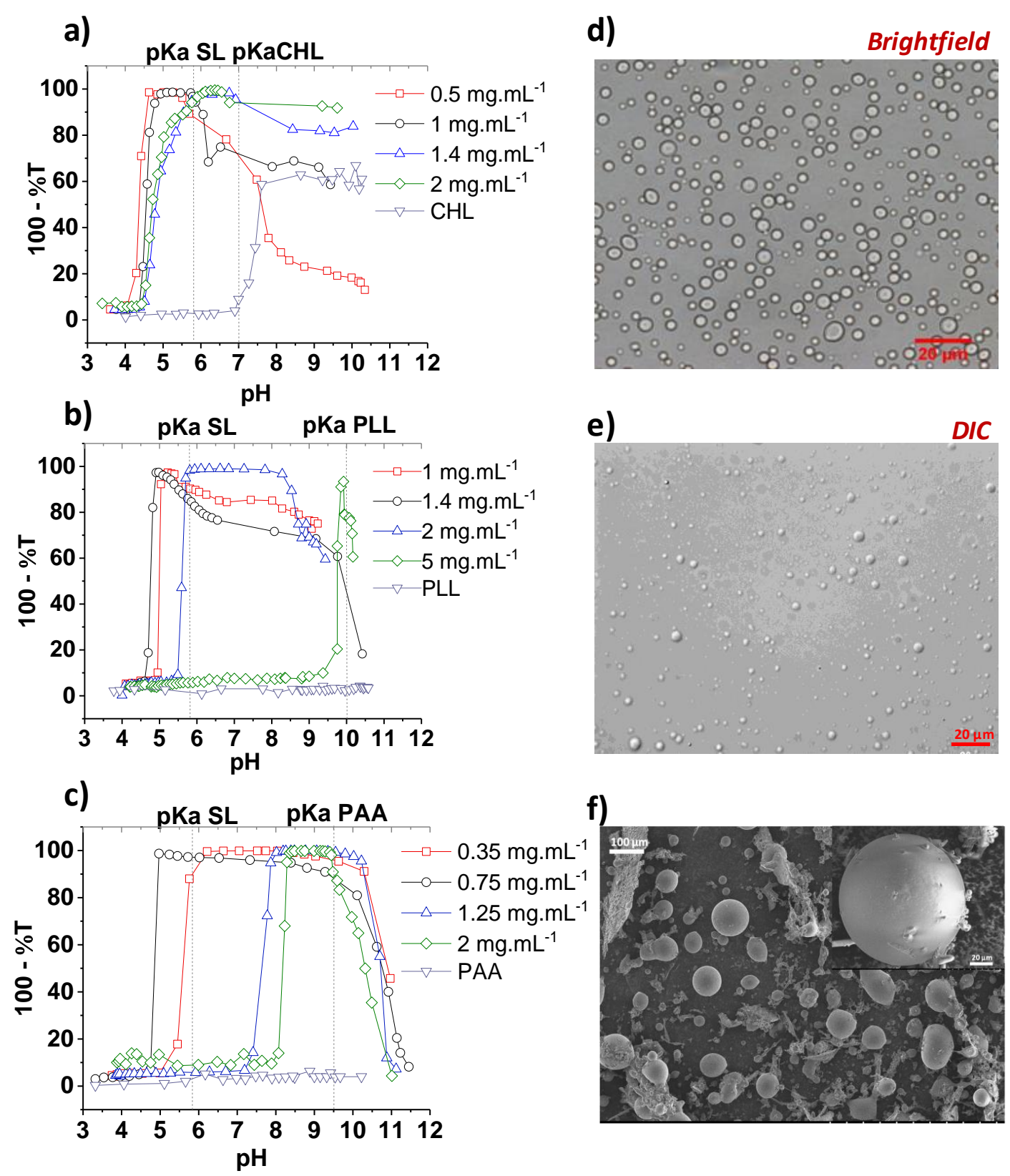

Figure 3 - Turbidity (100-\% T) as function of pH for the systems (a) SL-CHL, (b) SL-PLL and (c) SL-

PAA at different PEC concentrations and constant SL concentration $(5 \mathrm{mg} / \mathrm{mL})$ and $\mathrm{T}=23{ }^{\circ} \mathrm{C} .(\mathrm{d}-\mathrm{e})$

Optical microscope images of SL-CHL at pH 5.62 and SL-PAA at pH 7.64. (f) SEM images of the freeze dried SL-PLL coacervates at pH 7.

\section{Transition pH from a monophasic to a biphasic system}

Theoretically, the coacervation process should be favored in a condition of charge compensation between the negatively-charged SL and positively-charged PEC, that is above the pKa of SL and below the pKa of PEC. The PEC linear charge density and SL micelles surface charge density are controlled by the ionization degree of the terminal carboxylic and amino groups (Figure 1a). From the turbidimetric curves in Figure 3, it should be noticed that 
the width of the $\mathrm{pH}$ domain for a stable complex coacervate (Region 3) considerably depends on the type of PEC, and it can be ranked in the following order: CHL < PLL < PAA. The important difference between CHL and the other PEC could be related to the interval width between the SL pKa, which is close to $5.8,{ }^{63}$ and the pKa of the PEC, that is 7, 10 and 9.5 for CHL, PLL and PAA, respectively. Figure S 5 illustrates the influence of the pKa of PEC on the optimal coacervation region width (Figure S 1). However, the variation between PLL and PAA, the pKa values of which are very close, could be related to the employed concentrations of PLL (1-5 mg/mL) and PAA (0.35-2 mg/mL), and therefore to the SL-PEC stoichiometry. Moreover, the molecular weight of PAA $(\mathrm{Mw}=17.5 \mathrm{kDa})$ and PLL $(\mathrm{Mw} \approx 1-5 \mathrm{kDa})$ could also affect the coacervation. In fact, the increase of PEC chain length could enhance the coacervation process, as previously observed for complex coacervation between PEC and mixed micelles. ${ }^{43}$

$\mathrm{pH} \varphi$ is classically defined as the point of abrupt turbidity within a very small change in $\mathrm{pH}$ (Figure 2) and it is determined as the intercept of Region 1 and Region 2 in the turbidity curve (Figure S 6) and it has currently been interpreted as the $\mathrm{pH}$ of the appearance of turbidity, or visual phase separation. ${ }^{77}$ For a better understanding of the influence of PEC concentration, the $\mathrm{pH} \varphi$ was plotted against the reduced ratio (r'), introduced previously by Kaibara et al., ${ }^{77}$ and which is the result of the multiplication of the SL-PEC weight ratio by the factor [PEC monomer unit mass/SL molecular weight] (Figure 4). Since $r^{\prime}$ is related to the reciprocal number of charged sites of PEC per SL molecule, only the half of the CHL monomer unit mass was considered, since it possesses two functional amino-groups (Figure 1c).

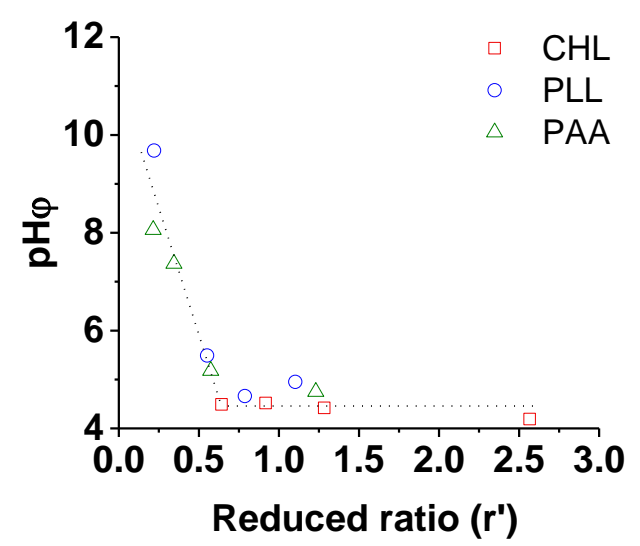

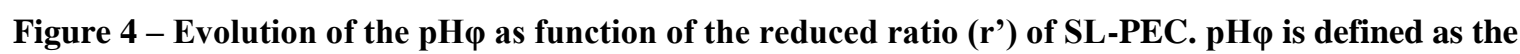
point of abrupt turbidity as indicated in Figure 2, while $r$ ' is the multiplication of the SL-PEC weight ratio by the factor [PEC monomer unit mass/SL molecular weight]. [SL] $=5 \mathrm{mg} / \mathrm{mL}$ 
CHL, PLL and PAA systems exhibit practically comparable plots, where the $\mathrm{pH} \varphi$ varies slightly for r' $\geq 0.6$ due to the saturation of PEC chains with the biosurfactant micelles but it increases sharply for $\mathrm{r}^{\prime}<0.6$, due to the possible existence of free PEC chains in the solution. This tendency could be generalized by expanding the studied SL-PEC ratios as previously performed for polymer/protein systems. ${ }^{86}$ But from Figure 4 it can be established that the evolution of $\mathrm{pH} \varphi$ as a function of the PEC type is minimized. This result suggests that the number of polymeric charged sites per SL micelles controls the coacervation process. Consequently, the structural differences of the PEC charged site had only a small effect on the phase separation, which is mainly driven by electrostatic interaction and it demonstrates the crucial role of $\mathrm{pH}$ by modulating the charge density and the strength of complexation. One should note that investigation of SL-CHL complex coacervation at lower reduced ratio $\left(\mathrm{r}^{\prime}<\right.$ 0.4 ), and where $\mathrm{pH} \varphi$ is expected to be higher than $\mathrm{pH} 7$, is not possible due to CHL insolubility in this $\mathrm{pH}$ range.

\section{Evolution of SL-PEC complexes with pH}

The complexation process of the SL biosurfactant with the different PEC was probed by monitoring the size evolution of SL-PEC complexes as function of $\mathrm{pH}$ by DLS measurements. The data are presented in Figure 5 in terms of hydrodynamic diameter as a function of the relative intensity.
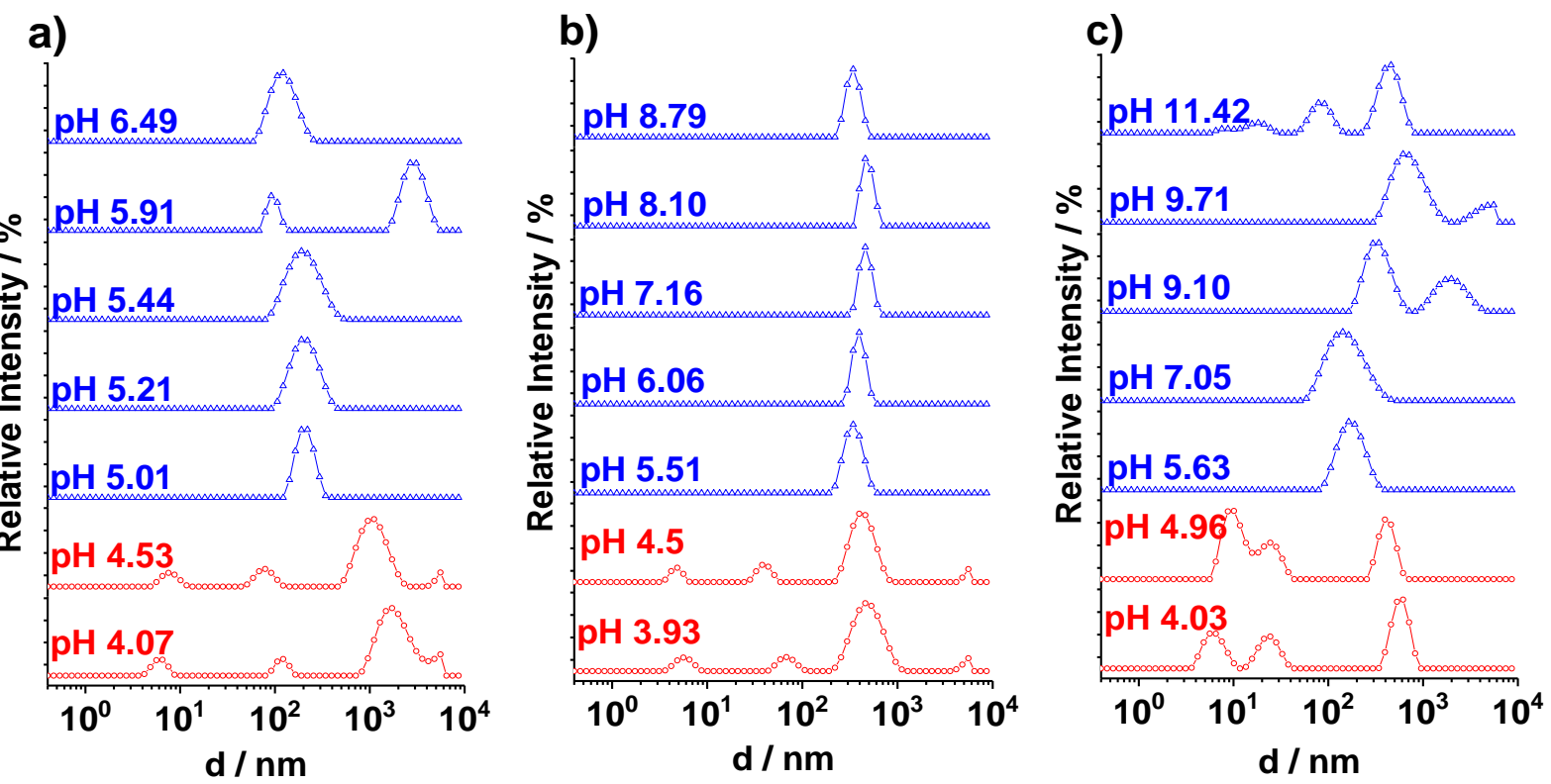

Figure 5 - Distribution of the hydrodynamic diameters of (a) SL-CHL, (b) SL-PLL and (c) SL-PAA complexe coacervates as a function of $\mathrm{pH}$ at $25^{\circ} \mathrm{C}$. [SL] $=5 \mathrm{mg} / \mathrm{mL},[\mathrm{CHL}]=1.4 \mathrm{mg} / \mathrm{mL},[\mathrm{PLL}]=2 \mathrm{mg} / \mathrm{mL}$, and $[$ PAA $]=0.75 \mathrm{mg} / \mathrm{mL}$. 
At $\mathrm{pH}<\mathrm{pH} \varphi($ Region 1 , here $\mathrm{pH}<5)$, all samples practically display three distinctive populations. The smallest diameter value is below $10 \mathrm{~nm}$, a size which is in agreement with the presence of free SL micelles. ${ }^{63}$ The size of the second and third populations vary with the SL-PEC system: SL-CHL (Figure 5a) shows two distributions at about $150 \mathrm{~nm}$ and $>1 \mu \mathrm{m}$

491

492

493

494

495

496

497

498

499

500

501

502

503

504

505

506

507

508

509

510

511

512

513

514

515

516

517

518

519

520 (beyond the limitation of the DLS instrument); SL-PLL (Figure 5b) at around $80 \mathrm{~nm} 500 \mathrm{~nm}$ while SL-PAA (Figure 5c) at about 25 and $600 \mathrm{~nm}$. Similar complexes were previously observed for mixed micelles and PEC mixtures during temperature-induced coacervation. Authors suggested that the coacervation process can be viewed as a clustering mechanism of soft colloidal particles which precedes coacervation. ${ }^{81,87}$ Indeed, soluble complexes from intra PEC-micelles and inter PEC-micelles are considered as precursors of coacervation. ${ }^{59}$ Nonetheless, it should be mentioned that relative number or volume size distributions only show free $\mathrm{SL}$ micelles at $\mathrm{pH}<\mathrm{pH} \varphi$ in Region 1, as shown in Figure S 7, thus indicating the majority of the sample is practically composed of free micelles.

The solution becomes cloudy above $\mathrm{pH} \varphi$ (Region 2 and Region 3), and the DLS measurements reveal the appearance of one single peak (hydrodynamic diameter> $100 \mathrm{~nm}$ ), the size of which is quite stable with $\mathrm{pH}$. These results reflect the formation of insoluble SLPEC complexes. By increasing the $\mathrm{pH}$, the size distribution of the complex coacervates shifts to larger aggregates for all SL-PEC complexes. Visually, the size of droplets increases and the solution becomes more opalescent due to coacervate coalescence and to the evolution from submicronic to microscopic droplets. When the $\mathrm{pH}$ is increased further in the vicinity or even beyond the PEC pKa, the hydrodynamic diameter becomes smaller again, as this can be observed for SL-CHL (Figure 5a) and SL-PAA (Figure 5c) in both the intensity and number/volume distributions (Figure S 7a,b). For instance, at pH 10.42 for the SL-PAA system, one can observe again that the majority of the population has a hydrodynamic diameter below $10 \mathrm{~nm}$ (Figure S 7a), suggesting the massive presence of micelles and the dissolution of the SL-PAA complex.

From DLS, one could suppose that at the starting $\mathrm{pH}(\mathrm{pH} \sim 4)$ in Region 1, the surface charge density of the SL micelles is strong enough to lead to soluble complexes formation with the different cationic PEC by Coulombic interaction. This seems to occur despite the very small amount of protonated $\mathrm{C}=\mathrm{O}$ groups, and in the absence of obvious screened Coulomb interaction among the micelles, otherwise observed at higher $\mathrm{pH}$ values by SAXS and SANS experiments. ${ }^{63,67,68}$ In this situation, the pH-induced complex coacervation mechanism takes place in four steps as follow: (i) formation of soluble complex from the free SL micelles and PEC chains at $\mathrm{pH} \sim 4$, (ii) the SL-PEC complexes become insoluble at $\mathrm{pH} \varphi$, 
when the interaction between both species becomes sufficiently high, (iii) precipitation when the interaction strength become too high and finally (iv) the dissolution of the precipitates. Combined electrophoretic mobility and turbidity data presented in Figure S 2a for SL-CHL and in Figure S 8 for SL-PAA show the strong correlation between the decrease of the electrophoretic mobility from $+2.4 \mu \mathrm{m} \mathrm{cm} / \mathrm{Vs}$ to zero, expected in a $\mathrm{pH}$ region rich in $\mathrm{NH}_{3}{ }^{+}$ groups, and the increase in turbidity, that is, complex formation. The coacervation is maximum for SL-CHL ( $\mathrm{P}_{\mathrm{CHL}}=0.25 \mathrm{mg} / \mathrm{mL}$ : low concentration) for an electrophoretic mobility of almost one; while all CHL chains are expected to be involved in the coacervates and are not expected to be free in solution (Figure S 2). Likewise for SL-PAA $\left(\mathrm{C}_{\mathrm{PAA}}=0.75\right.$ $\mathrm{mg} / \mathrm{mL}$ ), with a theoretical stoichiometry equals to one, the maximum coacervation region started significantly far from the point of electroneutrality, as also commented above. These observations are in line with the results reported in Figure $S 4 b$, where the maximum coacervation region was reported for charge ratios less than one. Theoretically, the absence of charge neutralization could be explained by the model proposed by Zhang and Shklovskii, ${ }^{88}$ who predicted that the oppositely charged macroions could form a neutral macroscopic drop by intracomplex, or intercomplex, disproportionation when the macroion charge stoichiometry deviates from unity.

\section{Quantification of the coacervation process}

The efficiency of coacervation and composition of coacervates are estimated by solution ${ }^{1} \mathrm{H}$ NMR, as detailed in the experimental section and presented in Figure S 9. In particular, the integral ratio between the resonances $\alpha-\mathrm{CH}_{2}\left(\mathrm{R}-\underline{\mathrm{C}}_{2}-\mathrm{C}=\mathrm{O}\right)$ of $\mathrm{SL}$ and $\alpha-\mathrm{CH}_{\mathrm{x}}\left(\mathrm{R}-\mathrm{C}_{\mathrm{z}}-\mathrm{NH}_{\mathrm{x}}, \mathrm{z}=\right.$ 1 for CHL, $\mathrm{z}=2$ for PAA, PLL) of the polyelectrolyte is used to quantify the $\mathrm{C}=\mathrm{O} / \mathrm{NH}_{\mathrm{x}}$ molar ratio in the coacervate $\left(\mathrm{C}=\mathrm{O}\right.$ and $\mathrm{NH}_{\mathrm{x}}$ refer to the carboxylic/carboxylate and amine/ammonium groups). On the contrary, since solution NMR is not sensitive to the sample involved in the coacervates, the loss in the spectral intensity after coacervation between $3 \mathrm{ppm}$ and $4 \mathrm{ppm}$ is used to quantify the extent of coacervation (residual method in Table 1b). The extent of coacervation is also verified by measuring the intensity loss (between $3 \mathrm{ppm}$ and 4 ppm) after recovery of the coacervate through centrifugation and disassembling it below the coacervation $\mathrm{pH}$ (centrifugation method in Table 1b).

Table 1a shows that the $\mathrm{C}=\mathrm{O} / \mathrm{NH}_{\mathrm{x}}$ molar ratio before and after coacervation stays practically unchanged, thus indicating that the fraction of SL and polyelectrolyte is stoichiometric in terms of charge pairing, as expected. In terms of the extent of coacervation (Table 1b), both 
methods used in this work nicely agree on the fact that about $25 \%$ of the initial polyelectrolyte-SL concentration is involved in the complex coacervation process. If a discrepancy seems to exist between the two methods for the SL-PAA system, we believe that incomplete recovery of coacervate during and after centrifugation is at its origin.

Table 1 - Quantification of the a) $\mathrm{C}=\mathrm{O} / \mathrm{NH}_{\mathrm{x}}$ molar ratio before $(\mathrm{pH}<5)$ and after $(\mathrm{pH}>5)$ coacervation (error is estimated to $10 \%$ ) and b) extent of coacervation, measured using two complementary methods. Centrifugation: the solution above $\mathrm{pH} 5$ is centrifuged to separate the coacervate, which is eventually redispersed in quantified. Residual: the amount of coacervate is estimated from the signal loss before and after coacervation $\mathrm{pH}$. All data are collected through solution ${ }^{1} \mathrm{H}$ NMR spectroscopy, which implied the use of a fully deuterated solvent $\left(\mathrm{D}_{2} \mathrm{O}\right)$. One car refer to Figure $\mathrm{S} 9$ for the typical NMR data.

\begin{tabular}{|c|c|c|}
\hline \multirow{2}{*}{ Polyelectrolyte/SL } & \multicolumn{2}{|c|}{$\mathrm{C}=\mathrm{O} / \mathrm{NH}_{\mathrm{x}}$ molar ratio } \\
\cline { 2 - 3 } & Before & After \\
\hline CHL & 0.8 & 1.0 \\
\hline PLL & 1.1 & 1.3 \\
\hline PAA & 0.7 & 0.5 \\
\hline
\end{tabular}

\begin{tabular}{|c|c|}
\hline \multicolumn{2}{|c|}{ Extent of coacervation (\%) } \\
\hline Centrifugation & Residual \\
\hline $24 \pm 1$ & $24 \pm 1$ \\
\hline $22 \pm 1$ & $23 \pm 2$ \\
\hline $27 \pm 2$ & $37 \pm 2$ \\
\hline
\end{tabular}

\section{Structure of the coacervates phase}

In order to investigate the structures of the complex coacervates during their formation, cryoTEM was carried out at different $\mathrm{pH}$-values for each SL-PEC system (Figure 6). The SL-CHL system at pH 5.94 (Region 3, Figure 6a,c) shows spherical particles of variable size (mainly of 50 and $200 \mathrm{~nm}$ ). These particles are homogeneous in texture and do not exhibit internal ordering or evidence of a particular organization. This type of structure is in a good agreement with coacervates observed in previous studies. ${ }^{22}$ Upon $\mathrm{pH}$ increasing to 6.33 (Region 3, Figure $6 \mathrm{~b}$ ), the structures keep the same size but they become denser, strongly contrasted, and lose their spherical shape. These aggregates could be generated by a dehydration phenomenon, which is associated to counterion expulsion and entropy loss, when SL-PEC interactions are promoted, as previously observed for polysaccharide-protein coacervation, ${ }^{89}$ and by analogy to complex coacervation of PEC-mixed micelles with temperature. ${ }^{90}$ For SLPLL at pH 5.77 (Figure 6d), at the Region 2/Region 3 frontier (Figure 6f), the coacervates exhibit also a spherical shape with a relative larger size (several hundred $\mathrm{nm}$ ); when the $\mathrm{pH}$ is increased to 7.38 (Region 3, Figure 6e,f), the droplets show, again, a higher apparent electron density. Finally, SL-PAA at pH 5.77 (Region 2, Figure 6g,i) displays discrete spherical particles in the $100 \mathrm{~nm}$ size and, in agreement with the other samples, denser particles at $\mathrm{pH}$ 6.83 (Region 3, Figure 6h,i). Interestingly, in practically all samples, a poorly-contrasted 
phase, most likely composed of free micelles and polymer, is observed at the frontier between Region 2 and Region 3. Coacervates in their initial stage of formation generally coexist with polymer and micelles, which seems the reason for the poor contrast, as suggested by the SLPLL system at pH 5.77 in Figure 6d, where the three spherical particles are superimposed to a broad continuum of matter. Once the $\mathrm{pH}$ defines Region 3, all samples show a much more contrasted, denser, phase composed of spheroidal particles; In this case, the background is much clearer, that is rich mainly composed of icy water and less rich in residual matter (polymer, micelles).
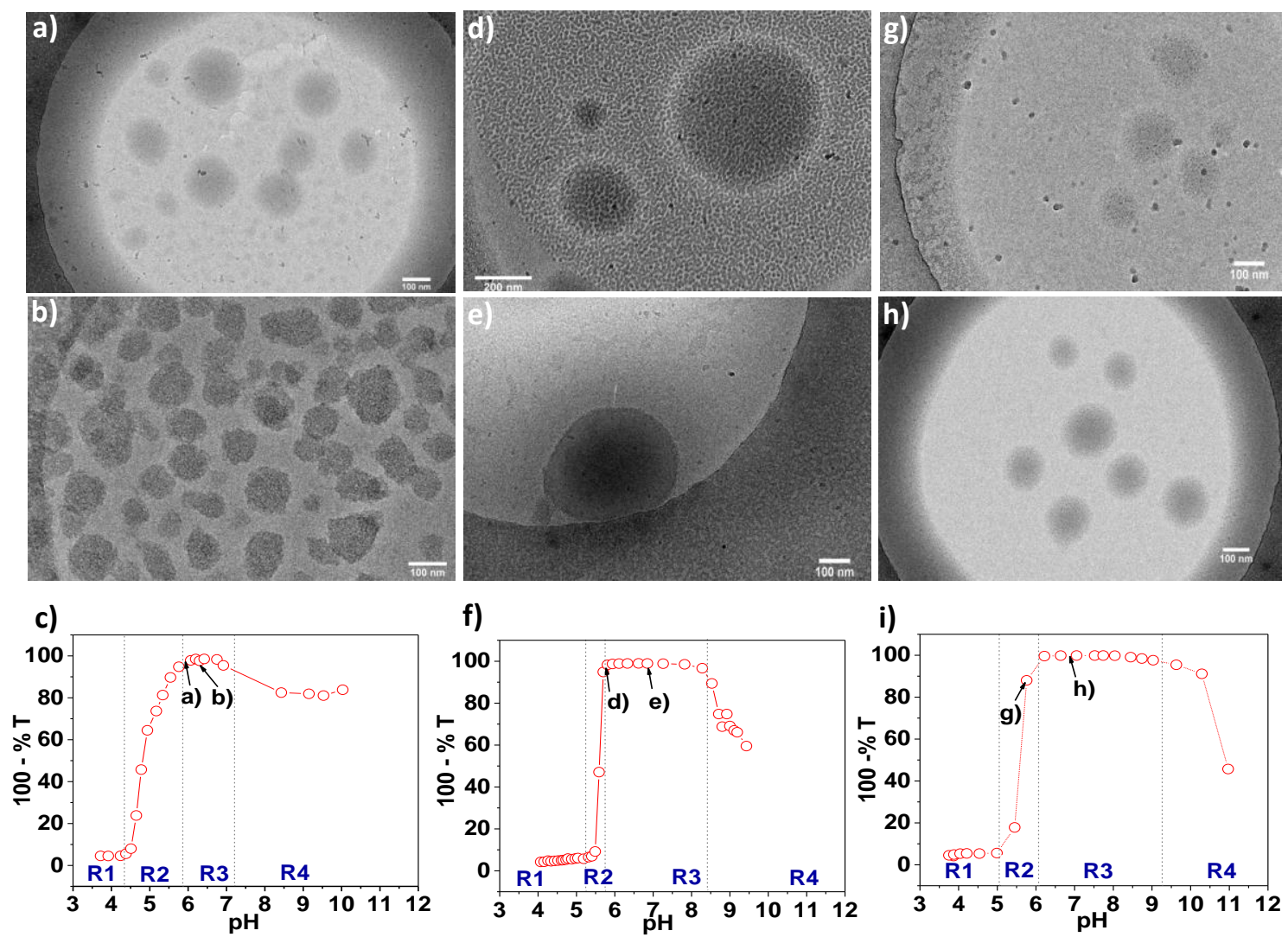

Figure 6 - Cryo-TEM images of SL-CHL coacervates at (a) pH 5.94 and (b) pH 6.33, SL-PLL coacervates at (d) pH 5.77 and (e) pH 7.38 and SL-PAA coacervates at (g) pH 5.77 and (h) pH 6.83. The corresponding turbidity of the observed SL-CHL, SL-PLL and SL-PAA systems is shown respectively in (c), (f) and (i). $[S L]=5 \mathrm{mg} / \mathrm{mL},[\mathrm{CHL}]=1.4 \mathrm{mg} / \mathrm{mL},[P L L]=2 \mathrm{mg} / \mathrm{mL}$, and $[\mathrm{PAA}]=0.75 \mathrm{mg} / \mathrm{mL}$. $R$ stands for Region.

Cryo-TEM shows that the coacervate structures critically depend on the $\mathrm{pH}$ and their evolution could be described as follow: at the early stage of coacervation, i.e, for $\mathrm{pH}$ just above the $\mathrm{pH} \varphi$ (Region 2) and until $\mathrm{pH}_{\max }$ (boundary between Region 2 and Region 3), spherical discrete droplets with a relatively low electron density are formed and are surrounded by a rich micellar phase. When $\mathrm{pH}>\mathrm{pH}_{\max }$, micelles gradually disappear due to 
their interactions to free PEC chains, as observed for SL-CHL at pH 5.94 and SL-PAA at pH 6.83. At later stages of coacervation, the droplets exhibit a more electron dense structure (e.g., SL-PLL at $\mathrm{pH}$ 7.38) due to the higher concentration of matter due to dehydration resulting from the release of counterions and water molecules from the molecular complex. The difference between the SL-PLL and SL-CHL structures is possibly related to the $\mathrm{pH}$ of the latter compared to the $\mathrm{pH}$ limit of coacervation (limitation between Region 3 and Region 4). In fact, SL-CHL and SL-PLL coacervates were respectively imaged at $\mathrm{pH} 6.33$ and $\mathrm{pH} 7.38$ while the $\mathrm{pH}$ limits are 6.92 and 8.53, respectively. Therefore SL-CHL coacervates were imaged at a later coacervation stage. Other parameters like the intrinsic molecular properties of CHL and PLL could also affect the fine coacervates structure, the description of which is out of the scope of this manuscript.

One should note that for SL-CHL system, the coexistence of both spherical coacervates and aggregates structures at $\mathrm{pH} 5.94$ and $\mathrm{pH} 6.33$ were detected (Figure S 9). The coacervate structures of SL-CHL, SL-PLL and SL-PAA at respectively pH 6.33, pH 7.38 and pH 6.83 are shown in Figure 7 at higher magnification with the corresponding Fast Fourrier Transform (FFT). One can notice that the internal organization depends strongly on the PEC. Moreover, the coacervates from SL-CHL and SL-PLL exhibit a well-defined interface compared to the SL-PAA. This observation is related to the coacervation stage. In fact SL-CHL and SL-PLL coacervates were imaged at $\mathrm{pH}$-values corresponding to $63 \%$ and $65 \%$ of the Region 3, while SL-PAA coacervates were imaged at only $24 \%$ of the optimal coacervation region. Further cryo-TEM images of SL-PEC coacervates at different $\mathrm{pH}$ are given in the electronic supplementary information (Figure S 10

Figure S 11, Figure S 12, Figure S 13).

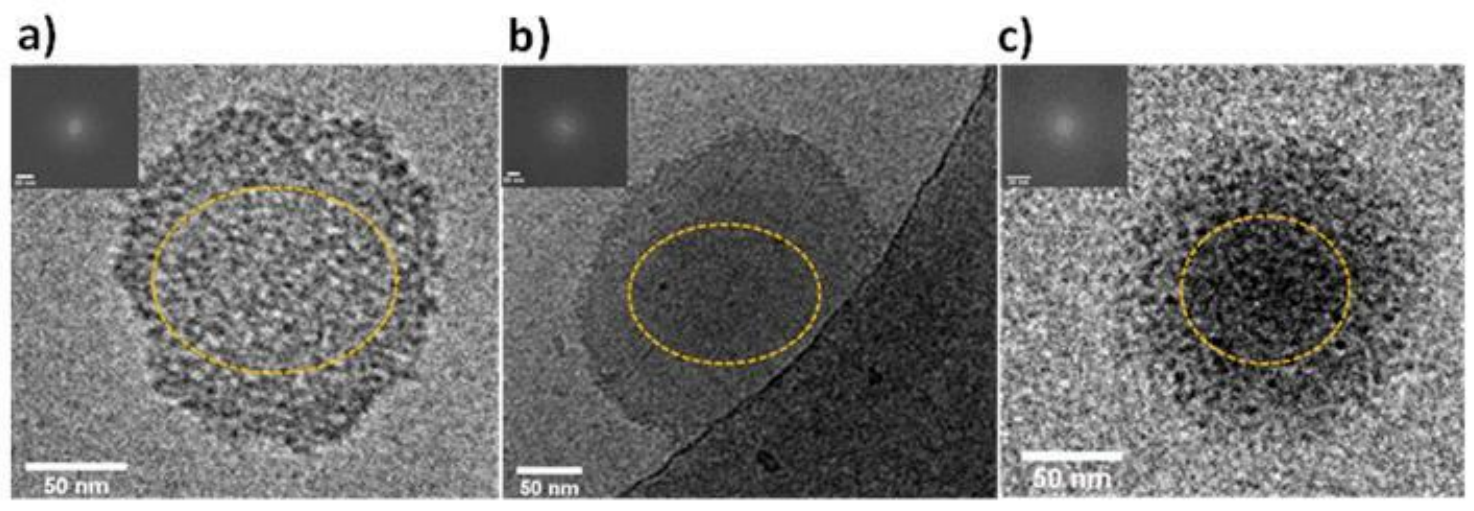

Figure 7 - Zoomed cryo-TEM images of complex coacervate particles of SL-CHL at (a) pH 6.33, (b) SLPLL at pH 7.38 and (c) SL-PAA at pH 6.83. $[\mathrm{SL}]=5 \mathrm{mg} / \mathrm{mL},[\mathrm{CHL}]=1.4 \mathrm{mg} / \mathrm{mL}$, $[\mathrm{PLL}]=2 \mathrm{mg} / \mathrm{mL}$, and $[P A A]=0.75 \mathrm{mg} / \mathrm{mL}$. 
633 To further investigate the structure of SL-PEC coacervates and their $\mathrm{pH}$ evolution, SAXS

634

635

636

637

638

639

640 measurements were conducted on SL-CHL and free CHL at different $\mathrm{pH}$ values (Figure 8), whereas SAXS/SANS data on SL micelles under similar conditions are reported elsewhere for comparison. ${ }^{63,67,68}$ In this study, the experimental q-range $0.05 \mathrm{~nm}^{-1}$ to $5 \mathrm{~nm}^{-1}$ is equivalent to sizes from $1.25 \mathrm{~nm}$ to $125 \mathrm{~nm}(\mathrm{~d} \approx 2 \pi / \mathrm{q})$, which is suitable for studying the organization of the coacervates phase but not their entire structure, since some coacervates are larger than 200 $\mathrm{nm}$, as previously shown by DLS, light microscopy and Cryo-TEM.
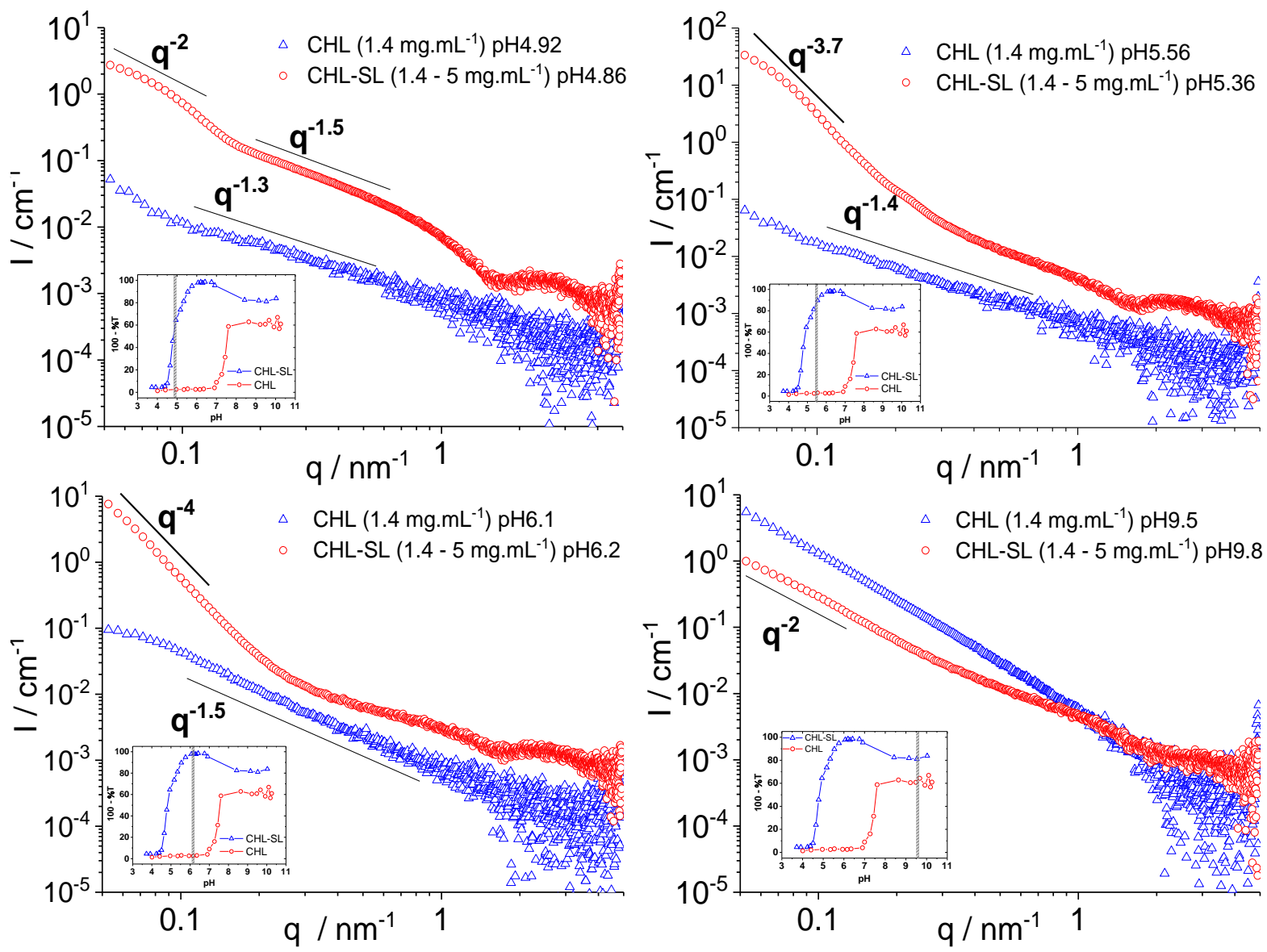

641

642

643

644

645

646

647

648

649

Figure 8 - SAXS profiles of CHL solution and SL-CHL mixtures at different pH-values. [SL]= $5 \mathrm{mg} / \mathrm{mL}$; $[\mathrm{CHL}]=1.4$ $\mathbf{m g} / \mathbf{m L}$

In the beginning of Region 2, below pH 5 (Figure 8a), SL-CHL is characterized by a scattering pattern typical of micelles in water for $\mathrm{q}>\sim 0.8 \mathrm{~nm}^{-1}$ and an increasing signal in the $\sim 0.1<\mathrm{q} / \mathrm{nm}^{-1}<\sim 0.8 \mathrm{~nm}^{-1}$ region (slope: -1.5 ), which most likely corresponds to the CHL contribution; its characteristic scattering profile recorded under similar conditions is given by the blue curve and it shows a slope of about -1.3. A further increase in the scattering signal for 
the SL-CHL system below $\mathrm{q} \sim 0.8 \mathrm{~nm}^{-1}$ indicates the presence of larger objects, thus confirming the intensity-weighted DLS data in Figure 5a, discussed previously. Considering the fact that free SL micelles in solution at acidic $\mathrm{pH}$ do not generally provide a pronounced low-q signal, ${ }^{91}$ one could attribute such a signal to either the free CHL (the SAXS profile of which in water also displays a small increase in the scattering signal at $\mathrm{q}<5 \mathrm{~nm}^{-1}$, Figure $8 \mathrm{a}$ ) or to what was hypothesized before, that is pre-formed SL-CHL complexes. However, such a contribution is small and, all in all, the SL-CHL pattern confirms that the system is mainly constituted by micelles and free polymer in solution, in agreement with the previous DLS data (Figure 5a, Figure S 7).

When the $\mathrm{pH}$ is set to be in the middle of Region 2 ( $\mathrm{pH} 5.56$, Figure 8b), the SL-CHL signal is still visible, although the low-q scattering contribution becomes more and more important. The low-q slope is close to -4 , which is expected in a scattering profile by a smooth interface, attributed to the coacervate droplet surface. As a comparison, the signal of free CHL has a slope of about -1.3 , which has practically not evolved in Region 3, when the formation of the complex coacervate is optimized, the SL-CHL SAXS pattern at $\mathrm{q}<0.2 \mathrm{~nm}^{-1}$ is again characterized by a frank -4 slope, while the high-q portion of the curve reflects the core of the coacervate assembly (Figure 8c). Although the interpretation is not straightforward, one can still observe the presence of an oscillation characteristics of a micellar form factor at $\mathrm{q}>1 \mathrm{~nm}^{-}$ ${ }^{1}$, showing that the micellar structure is kept intact within the coacervate. The stability of SL micelles during their binding to PEC comes from the absence of change of the solubilizing capacity of micelles. ${ }^{92}$ Again, the scattering pattern of free CHL in the same $\mathrm{pH}$ range is not comparable with the coacervate signal and it has not evolved since acidic $\mathrm{pH}$ : the Porod exponent of CHL ranges between -1.3 and -1.5 . Generally, a Porod exponent of $\approx-1$ refers to a rod-like structure, although deviations from a -1 power law may occur and they can be explained by deviations of polymer chain linearity due to, e.g., intra-chain electrostatic repulsion affecting the expected rigid rod-like chain conformation for a semi-flexible polymer like CHL. One should note that the Porod exponent found here is close to $-5 / 3$, which is generally attributed to the scattering profile of a swollen chain, corresponding to a polymer in a good solvent, ${ }^{93,94}$ which is the case of CHL in water below pH 7. Finally, well above the pKa of CHL ( $\mathrm{pH}>9.5$, Region 4), the signal of both CHL and SL-CHL are now comparable at $\mathrm{q}<\sim 0.8 \mathrm{~nm}^{-1}$, both characterized by a Porod exponent of -2 , while one can still observe a mild contribution of the micelles to the SAXS signal above $\mathrm{q} \sim 0.8 \mathrm{~nm}^{-1}$. The different oscillating profile of SAXS signal for SL micelles observed at acidic and basic $\mathrm{pH}\left(\mathrm{q}>\sim 0.8 \mathrm{~nm}^{-1}\right)$ should 
not be surprising because it has been observed before and described as a difference in terms of neutral and charged SL arrangement within the micelle itself. ${ }^{91}$ In the end, the SAXS analysis shows that in Region 4 the system is composed of the free CHL polymer and in a Gaussian chain conformation, ${ }^{93,94}$ and free charged SL micelles, as hypothesized above.

\section{Conclusions}

In this work, we demonstrate the ability of biobased sophorolipid bolaform biosurfactant micelles to form complex coacervates with different cationic polyelectrolytes, i.e. a naturally derived oligosaccharide and two synthetic polymers. The coacervation process is mainly driven by $\mathrm{pH}$ and turbidimetric titration revealed that the coacervates can be formed in a large $\mathrm{pH}$ range as function of the cationic polyelectrolyte type and concentration. The charge-pairing mechanism is confirmed by quantitative NMR analysis, which also shows that $25 \%$ of the initial SL-polyelectrolyte concentration is involved in the coacervates.

The coacervation structure investigated by cryo-TEM shows the coexistence of polymer and micelles upon coacervate formation and the presence of well-defined coacervates in their stability region. Cryo-TEM suggests that micelles compose the coacervate and this piece of evidence is confirmed by SAXS experiments, which show that micelles and free polymer coexist and probably interact out of the coacervate-formation window. SAXS also shows that coacervates are themselves composed of micelles. This description of the complex coacervate formation between a chargeable bolaform surfactant and chargeable polyelectrolytes is consistent with what has been described for more classical ionic surfactants-polyelectrolyte systems.

Finally, this study offers new prospects for the use of bolaform sophorolipid micelles to prepare complex coacervates which could be useful for pollutants and dye removal ${ }^{54,55}$ or like an encapsulation matrix for drug delivery applications. In a general view, the valorization of such bolaamphiphile molecules through the investigation of their binding behavior to further macromolecules seems to be a promising approach to prepare future functional soft materials.

\section{Acknowledgments}

This work received financial support by the European Synchrotron Radiation Facility (ESRF), Grenoble, France, under the experiment number MX1821. The research leading to these results has received funding from the European Community's Seventh Framework Programme (FP7/2007-2013) under Grant Agreement n Biosurfing/289219. Dr. Corinne Gerardin (Institut Charles Gerhardt, Montpellier, France) for inspiring discussions. 


\section{References}

7171 W. Von Rybinski and K. Hill, Angew. Chemie - Int. Ed., 1998, 37, 1328-1345.

7182 A.-S. Cuvier, J. Berton, C. V Stevens, G. C. Fadda, F. Babonneau, I. N. a Van Bogaert, W. Soetaert, G. Pehau-Arnaudet and N. Baccile, Soft Matter, 2014, 10, 3950-9. K. Hill and C. le Hen-Ferrenbach, in Sugar-Based Surfactants: Fundamentals and Applications, ed. C. C. Ruiz, Boca Raton, CRC Press., 2009, pp. 1-20.

4 K. Valappil Sajna, R. Höfer, R. K. Sukumaran, L. Devi Gottumukkala and A. Pandey, in Industrial Biorefineries and White Biotechnology, eds. A. Pandey, R. Höfer, C. Larroche, M. Taherzadeh and K. M. Nampoothiri, Elsevier, Amsterdam, Oxford, Waltham, 1st edn., 2015, pp. 499-521.

5 U. Rau, S. Hammen, R. Heckmann, V. Wray and S. Lang, Ind. Crops Prod., 2001, 13, 8592.

6 D. W. G. Develter and S. J. J. Fleurackers, in Surfactants from Renewable Resources, John Wiley \& Sons, Ltd, 2010, pp. 213-238.

7 M. R. de Oliveira, D. Camilios-Neto, C. Baldo, A. Magri and M. A. P. Colabone Celligoi, Int. J. Sci. Technol. Res., 2014, 3, 133-143.

8 I. N. A. Van Bogaert, K. Saerens, C. De Muynck, D. Develter, W. Soetaert and E. J. Vandamme, Appl. Microbiol. Biotechnol., 2007, 76, 23-34.

91995.

10 S. L. Fu, S. R. Wallner, W. B. Bowne, M. D. Hagler, M. E. Zenilman, R. Gross and M. H. Bluth, J. Surg. Res., 2008, 148, 77-82.

11 N. Baccile, N. Nassif, L. Malfatti, I. N. a. Van Bogaert, W. Soetaert, G. Pehau-Arnaudet and F. Babonneau, Green Chem., 2010, 12, 1564.

12 N. Baccile, F. Babonneau, I. M. Banat, K. Ciesielska, A.-S. Cuvier, B. Devreese, B. Everaert, H. Lydon, R. Marchant, C. A. Mitchell, S. Roelants, L. Six, E. Theeuwes, G. Tsatsos, G. E. Tsotsou, B. Vanlerberghe, I. N. A. Van Bogaert and W. Soetaert, ACS Sustain. Chem. Eng., 2017, 5, 1186-1198.

13 P. Dubey, S. Kumar, V. K. Aswal, S. Ravindranathan, P. R. Rajamohanan, A. Prabhune and A. Nisal, Biomacromolecules, 2016, 17, 3318-3327.

14 J. K. Madsen, J. D. Kaspersen, C. B. Andersen, J. Nedergaard Pedersen, K. K. Andersen, J. S. Pedersen and D. E. Otzen, Biochemistry, 2017, 56, 4256-4268.

15 E. Guzmán, S. Llamas, A. Maestro, L. Fernández-Peña, A. Akanno, R. Miller, F. Ortega and R. G. Rubio, Adv. Colloid Interface Sci., 2015, 233, 38-64.

16 L. Chiappisi, S. Prévost, I. Grillo and M. Gradzielski, Langmuir, 2014, 30, 1778-1787.

17 H. G. Bungenberg de Jong and H. R. Kruyt, Proc.Acad.Sci Amsterdam, 1929, 32, 849856.

18 F. M. Menger and B. M. Sykes, Langmuir, 1998, 14, 4131-4137.

19 F. M. Menger, A. V Peresypkin, K. L. Caran and R. P. Apkarian, Langmuir, 2000, 16, 9113-9116.

20 H. B. Bohidar, J. Surf. Sci. Technol., 2008, 24, 105-124.

21 A. I. Oparin, Dover Publ. New York, 1953.

22 S. Koga, D. S. Williams, A. W. Perriman and S. Mann, Nat. Chem., 2011, 3, 720-4.

23 T.-Y. Dora Tang, C. Rohaida Che Hak, A. J. Thompson, M. K. Kuimova, D. S. Williams, A. W. Perriman and S. Mann, Nat. Chem., 2014, 6, 527-533.

24 W. M. Aumiller and C. D. Keating, Nat. Chem., 2015.

25 C. P. Brangwynne, P. Tompa and R. V. Pappu, Nat. Phys., 2015, 11, 899-904.

26 A. Aguzzi and M. Altmeyer, Trends Cell Biol., 2016, 26, 547-558. 
F. W. Tiebackx, Zeitschrift fur Chemie und Ind. der Kolloide, 1911, 8, 198-201.

B. Mohanty and H. B. Bohidar, Biomacromolecules, 2003, 4, 1080-1086.

R. Wang, M. Tian and Y. Wang, Soft Matter, 2014, 10, 1705-1713.

M. Wang, Y. Fan, Y. Han, Z. Nie and Y. Wang, Langmuir, 2013, 29, 14839-14847.

M. G. Khaledi, S. I. Jenkins and S. Liang, Langmuir, 2013, 29, 2458-2464.

A. B. Kayitmazer, A. F. Koksal and E. Kilic lyilik, Soft Matter, 2015, 11, 8605-8612.

33 H. Espinosa-andrews, J. G. Ba, F. Cruz-sosa and E. J. Vernon-carter, Biomacromolecules, 2007, 8, 1313-1318.

34 Q. Wang and J. B. Schlenoff, Macromolecules, 2014, 47, 3108-3116.

35 A. Boudier, A. Aubert-Pouëssel, C. Gérardin, J. M. Devoisselle and S. Bégu, Int. J. Pharm., 2009, 379, 212-217.

36 J. Warnant, N. Marcotte, J. Reboul, G. Layrac, A. Aqil, C. Jerôme, D. A. Lerner and C. Gérardin, Anal. Bioanal. Chem., 2012, 403, 1395-1404.

37 J. Reboul, T. Nugay, N. Anik, H. Cottet, V. Ponsinet, M. In, P. Lacroix-Desmazes and C. Gérardin, Soft Matter, 2011, 7, 5836.

38 C. G. De Kruif, F. Weinbreck and R. De Vries, Curr. Opin. Colloid Interface Sci., 2004, 9, 340-349.

39 C. Schmitt and S. L. Turgeon, Adv. Colloid Interface Sci., 2011, 167, 63-70.

40 L. Aberkane, J. Jasniewski, C. Gaiani, J. Scher and C. Sanchez, Langmuir, 2010, 26, 12523-12533.

41 D. Leisner and T. Imae, J. Phys. Chem. B, 2003, 107, 8078-8087.

42 Y. Wang, K. Kimura, Q. Huang, P. L. Dubin and W. Jaeger, Macromolecules, 1999, 32, $7128-7134$.

43 Y. Wang, K. Kimura, P. L. Dubin and W. Jaeger, Macromolecules, 2000, 33, 3324-3331.

44 S. Mukherjee, A. Dan, S. C. Bhattacharya, A. K. Panda and S. P. Moulik, Langmuir, 2011, 27, 5222-5233.

45 Y. J. Li, J. L. Xia and P. L. Dubin, Macromolelcules, 1994, 27, 7049-7055.

46 Y. J. Li, P. L. Dubin, H. Dautzenberg, U. Luck, J. Hartmann and Z. Tuzar, Macromolelcules, 1995, 28, 6795-6798.

47 P. L. Dubin and D. Davis, Colloids and Surfaces, 1985, 13, 113-124.

48 B. D. Winslow, H. Shao, R. J. Stewart and P. A. Tresco, Biomaterials, 2010, 31, 93739381.

49 H. Chu, J. Gao, C.-W. Chen, J. Huard and Y. Wang, Proc. Natl. Acad. Sci. U. S. A., 2011, 108, 13444-9.

50 N. R. Johnson and Y. Wang, Expert Opin Drug Deliv, 2014, 11, 1829-1832.

51 D. S. Hwang, H. Zeng, A. Srivastava, D. V Krogstad, M. Tirrell, J. N. Israelachvili and J. H. Waite, Soft Matter, 2010, 6, 3232.

52 S. Kim, H. Y. Yoo, J. Huang, Y. Lee, S. Park, Y. Park, S. Jin, Y. M. Jung, H. Zeng, D. S. Hwang and Y. Jho, ACS Nano, 2017, 11, 6764-6772.

53 N. Baccile, J. Reboul, B. Blanc, B. Coq, P. Lacroix-Desmazes, M. In and C. Gérardin, Angew. Chemie - Int. Ed., 2008, 47, 8433-8437.

54 L. Chiappisi, M. Simon and M. Gradzielski, ACS Appl. Mater. Interfaces, 2015, 7, 61396145.

55 W. Zhao, Y. Fan, H. Wang and Y. Wang, Langmuir, 2017, 33, 6846-6856.

56 W. Zhao and Y. Wang, Adv. Colloid Interface Sci., 2017, 199-212.

57 C. D. Bain, P. M. Claesson, D. Langevin, R. Meszaros, T. Nylander, C. Stubenrauch, S. Titmuss and R. von Klitzing, Adv. Colloid Interface Sci., 2010, 155, 32-49. 
81058 P. L. Dubin, C. H. Chew and L. M. Gan, J. Colloid Interface Sci., 1989, 128, 566-576.

81159 E. Kizilay, A. B. Kayitmazer and P. L. Dubin, Adv. Colloid Interface Sci., 2011, 167, 24-

812

813

814

815

816

817

818

819

820

821

822

823

824

825

826

827

828

829

830

831

832

833

834

835

836

837

838

839

840

841

842

843

844

845

846

847

848

849

850

851

852

853

854

855

856 37.

60 C. L. Cooper, A. Goulding, A. B. Kayitmazer, S. Ulrich, S. Stoll, S. Turksen, S. I. Yusa, A. Kumar and P. L. Dubin, Biomacromolecules, 2006, 7, 1025-1035.

61 A. B. Kayitmazer, Adv. Colloid Interface Sci., 2016, 239, 169-177.

62 T. Imura, H. Yanagishita and D. Kitamoto, J. Am. Chem. Soc., 2004, 126, 10804-10805.

63 S. Manet, A. S. Cuvier, C. Valotteau, G. C. Fadda, J. Perez, E. Karakas, S. Abel and N. Baccile, J. Phys. Chem. B, 2015, 119, 13113-13133.

64 S. Pillai, K. S.. Paul, W and C. P., Prog. Polym. Sci., 2009, 34, 641-678.

65 S. K. Samal, M. Dash, S. Van Vlierberghe, D. L. Kaplan, E. Chiellini, C. van Blitterswijk, L. Moroni and P. Dubruel, Chem. Soc. Rev., 2012, 41, 7147.

66 N. Baccile, A. S. Cuvier, C. Valotteau and I. N. A. Van Bogaert, Eur. J. Lipid Sci. Technol., 2013, 115, 1404-1412.

67 N. Baccile, F. Babonneau, J. Jestin, G. Pehau-Arnaudet and I. Van Bogaert, ACS Nano, 2012, 6, 4763-4776.

68 N. Baccile, J. S. Pedersen, G. Pehau-Arnaudet and I. N. A. Van Bogaert, Soft Matter, 2013, 9, 4911.

69 P. Dhasaiyan, P. Le Griel, S. Roelants, E. Redant, I. N. A. Van Bogaert, S. Prevost, B. L. V. Prasad and N. Baccile, ChemPhysChem, 2017, 18, 643-652.

70 W. Liu, S. Sun, Z. Cao, X. Zhang, K. Yao, W. W. Lu and K. D. K. Luk, Biomaterials, 2005, 26, 2705-2711.

71 S. R. Lewis, S. Datta, M. Gui, E. L. Coker, F. E. Huggins, S. Daunert, L. Bachas and D. Bhattacharyya, Proc. Natl. Acad. Sci., 2011, 108, 8577-8582.

72 H. Ohshima, Colloid Polym. Sci., 2007, 285, 1411-1421.

73 P. Pernot, A. Round, R. Barrett, A. De Maria Antolinos, A. Gobbo, E. Gordon, J. Huet, J. Kieffer, M. Lentini, M. Mattenet, C. Morawe, C. Mueller-Dieckmann, S. Ohlsson, W. Schmid, J. Surr, P. Theveneau, L. Zerrad and S. McSweeney, J. Synchrotron Radiat., 2013, 20, 660-664.

74 A. Round, F. Felisaz, L. Fodinger, A. Gobbo, J. Huet, C. Villard, C. E. Blanchet, P. Pernot, S. McSweeney, M. Roessle, D. I. Svergun and F. Cipriani, Acta Crystallogr. Sect. D Biol. Crystallogr., 2015, 71, 67-75.

75 G. Ashiotis, A. Deschildre, Z. Nawaz, J. P. Wright, D. Karkoulis, F. E. Picca and J. Kieffer, J. Appl. Crystallogr., 2015, 48, 510-519.

76 H. Espinosa-andrews, J. G. Baéz-Gonzalez, F. Cruz-sosa and E. J. Vernon-carter, Biomacromolecules, 2007, 8, 1313-1318.

77 K. Kaibara, T. Okazaki, H. B. Bohidar and P. L. Dubin, Biomacromolecules, 2000, 1, 100107.

78 F. Weinbreck, R. de Vries, P. Schrooyen and C. G. de Kruif, Biomacromolecules, 2003, 4, 293-303.

79 C. Sanchez, G. Mekhloufi and D. Renard, J. Colloid Interface Sci., 2006, 299, 867-873.

80 K. Yoshida and P. L. Dubin, in Colloids and Surfaces A: Physicochemical and Engineering Aspects, 1999, vol. 147, pp. 161-167.

81 E. Kizilay, S. Maccarrone, E. Foun, A. D. Dinsmore and P. L. Dubin, J. Phys. Chem. B, 2011, 115, 7256-7263.

82 X. Wang, J. Wang, Y. Wang and H. Yan, Langmuir, 2004, 20, 9014-9018.

83 P. L. Flory, J. Chem. Phys., 1942, 10, 51-61. 
85784 M. L. Huggins, J. Am. Chem. Soc., 1942, 64, 1712-1719.

85885 D. R. Rigsbee and P. L. Dubin, Langmuir, 1996, 7, 1928-1929.

85986 K. Kaibara, T. Okazaki, H. B. Bohidar and P. L. Dubin, Biomacromolecules, 2000, 1, 100$860 \quad 107$.

86187 E. Kizilay, A. D. Dinsmore, D. A. Hoagland, L. Sun and P. L. Dubin, Soft Matter, 2013, 9, 8627320.

86388 R. Zhang and B. I. Shklovskii, Phys. A Stat. Mech. its Appl., 2005, 352, 216-238.

86489 A. B. Kayitmazer, S. P. Strand, C. Tribet, W. Jaeger and P. L. Dubin, Biomacromolecules, $865 \quad 2007,8,3568-3577$.

86690 M. W. Liberatore, N. B. Wyatt, M. Henry, P. L. Dubin and E. Foun, Langmuir, 2009, 25,

867

868

869

870

871

872 13376-13383.

91 N. Baccile, A. S. Cuvier, S. Prévost, C. V. Stevens, E. Delbeke, J. Berton, W. Soetaert, I.

N. A. Van Bogaert and S. Roelants, Langmuir, 2016, 32, 10881-10894.

92 P. L. Dubin, J. H. Gruber, J. Xia and H. Zhang, J. Colloid Interface Sci., 1992, 148, 35-41.

93 J. Teixera, J. Appl. Crystallogr., 1988, 21, 781-785.

873

874

875

876

877

878

879

880

881

882

883

884

885

886 
3 Complex coacervation of natural sophorolipid bolaamphiphile 4 micelles with cationic polyelectrolytes

5 Ghazi Ben Messaoud, ${ }^{\mathrm{a}}$ Lyndsay Promeneur, ${ }^{\mathrm{a}}$ Martha Brennich, ${ }^{\mathrm{b}}$ Sophie Roelants, ${ }^{\mathrm{c}, \mathrm{d}}$ Patrick 6 Le Griel, ${ }^{\mathrm{a}}$ Niki Baccile ${ }^{\mathrm{a}, *}$

7

8 a Sorbonne Université, Centre National de la Recherche Scientifique, Laboratoire de Chimie 9 de la Matière Condensée de Paris, LCMCP, F-75005 Paris, France ${ }^{\mathrm{b}}$ European Molecular Biology Laboratory, Synchrotron Crystallography Group, 71 Avenue des Martyrs, 38042 Grenoble, France. Links 653, Ghent, Oost-Vlaanderen, BE 9000 


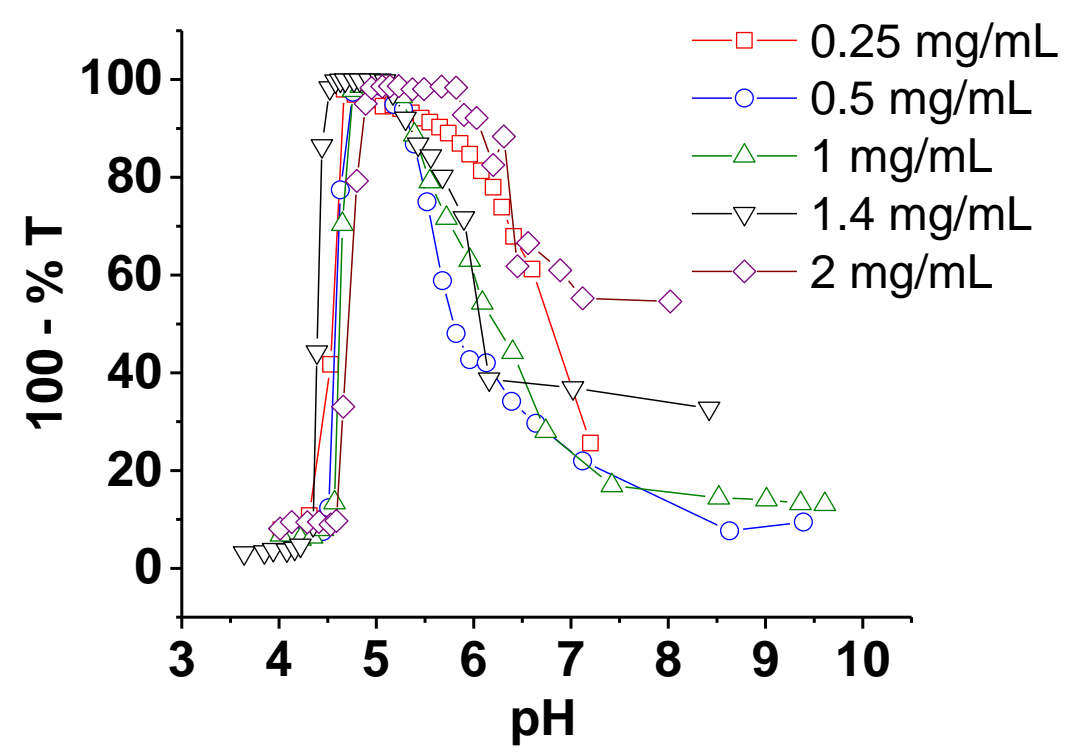

25

26 Figure S 1 - Evolution of turbidity during coacervation of SL micelles ([SL]= $10 \mathrm{mg} / \mathrm{mL}$ ) with CHL

$27([\mathrm{CHL}]=0.25-2 \mathrm{mg} / \mathrm{mL})$ as function of $\mathrm{pH}$. 
a)

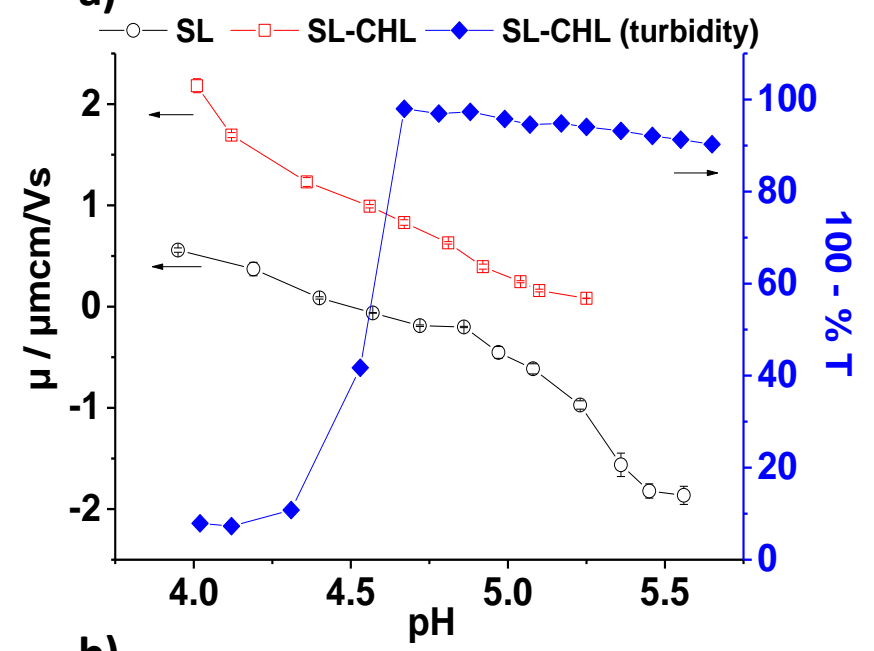

b)

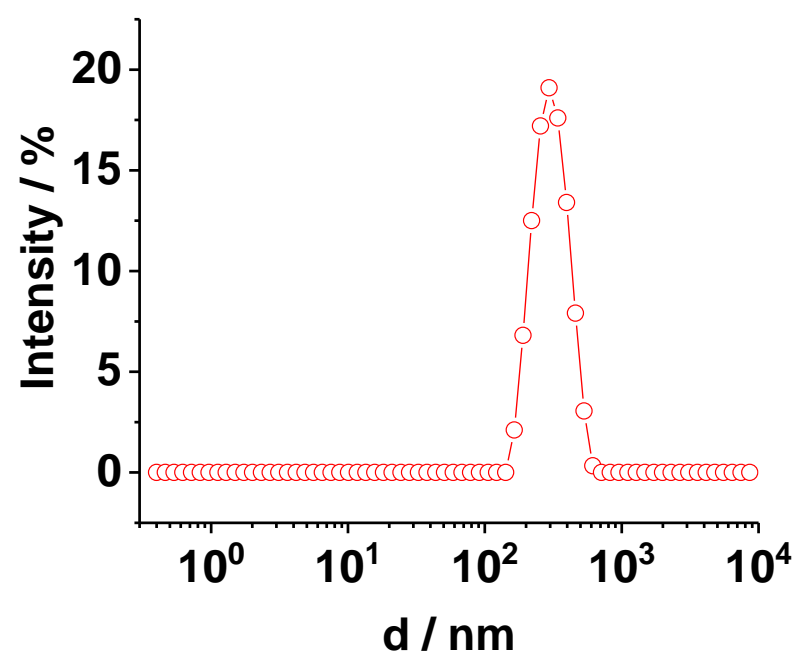

c)

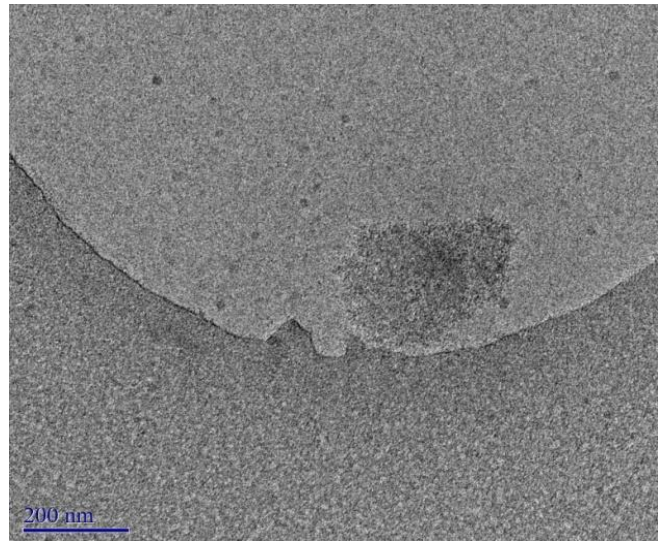

d)

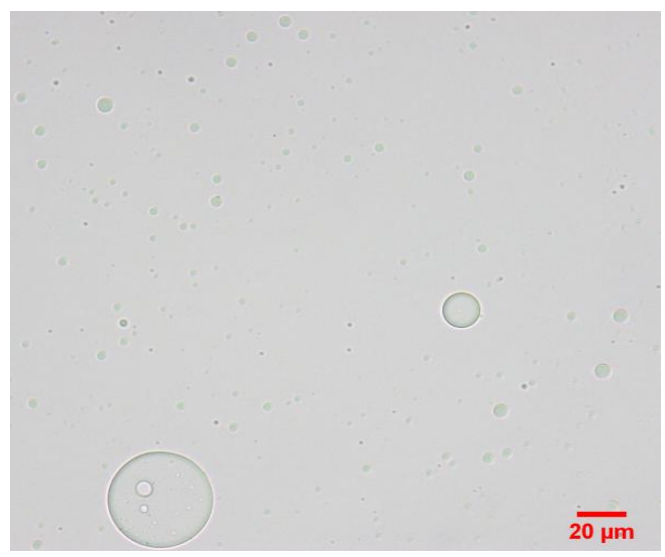

29 

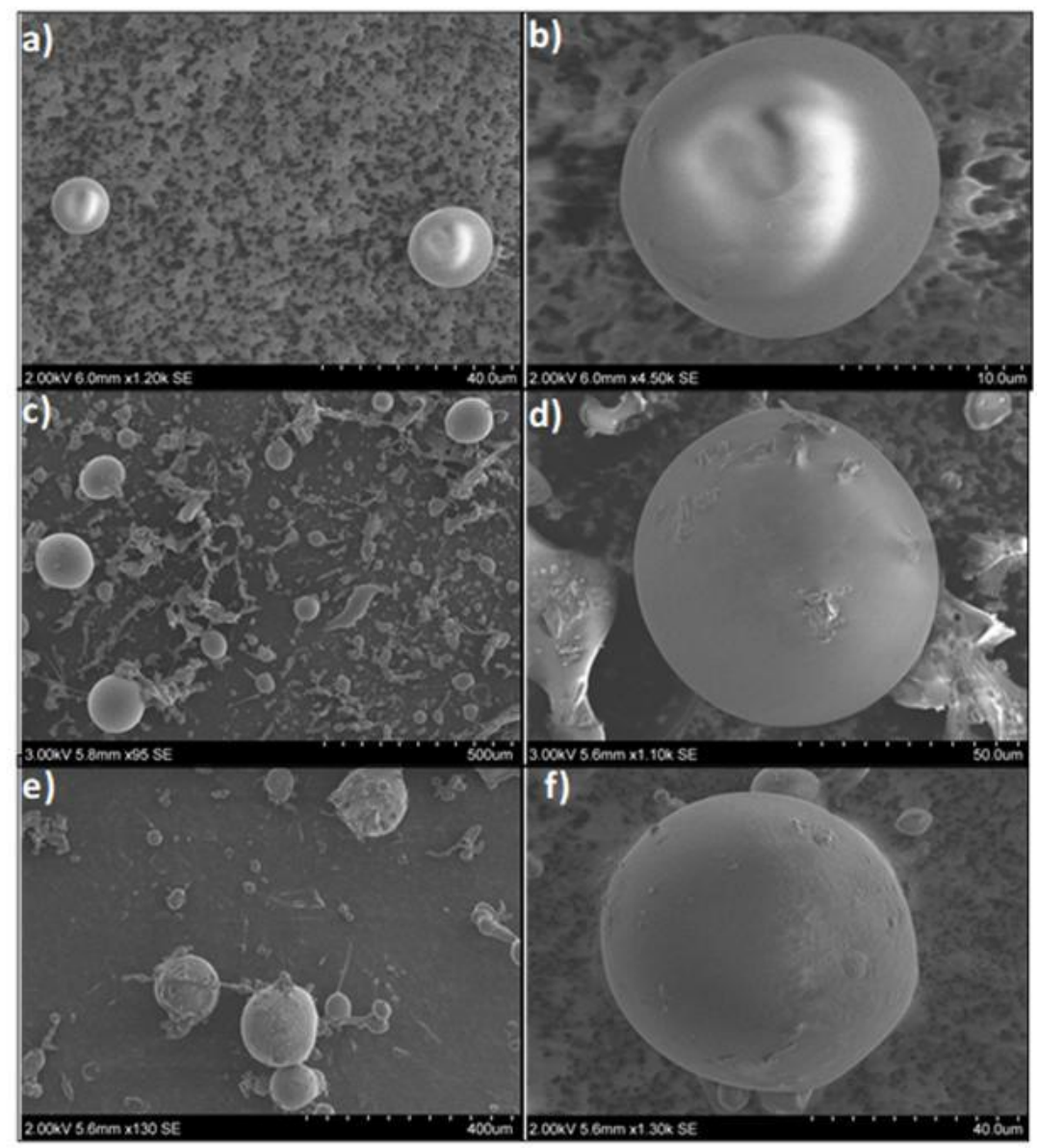

39 Figure 33 - SEM images of (a,b) SL-CHL ([CHL]= $1.4 \mathrm{mg} / \mathrm{mL}, \mathrm{pH}$ 6.2), (c,d) SL-PLL ([PLL]= $2 \mathrm{mg} / \mathrm{mL}$, $40 \mathrm{pH} \mathrm{7}$ ) and (e,f) SL-PAA ([PAA] $=0.75 \mathrm{mg} / \mathrm{mL}, \mathbf{p H} 8$ ) complex coacervates prepared with $[\mathrm{SL}]=5 \mathrm{mg} / \mathrm{mL}$. 

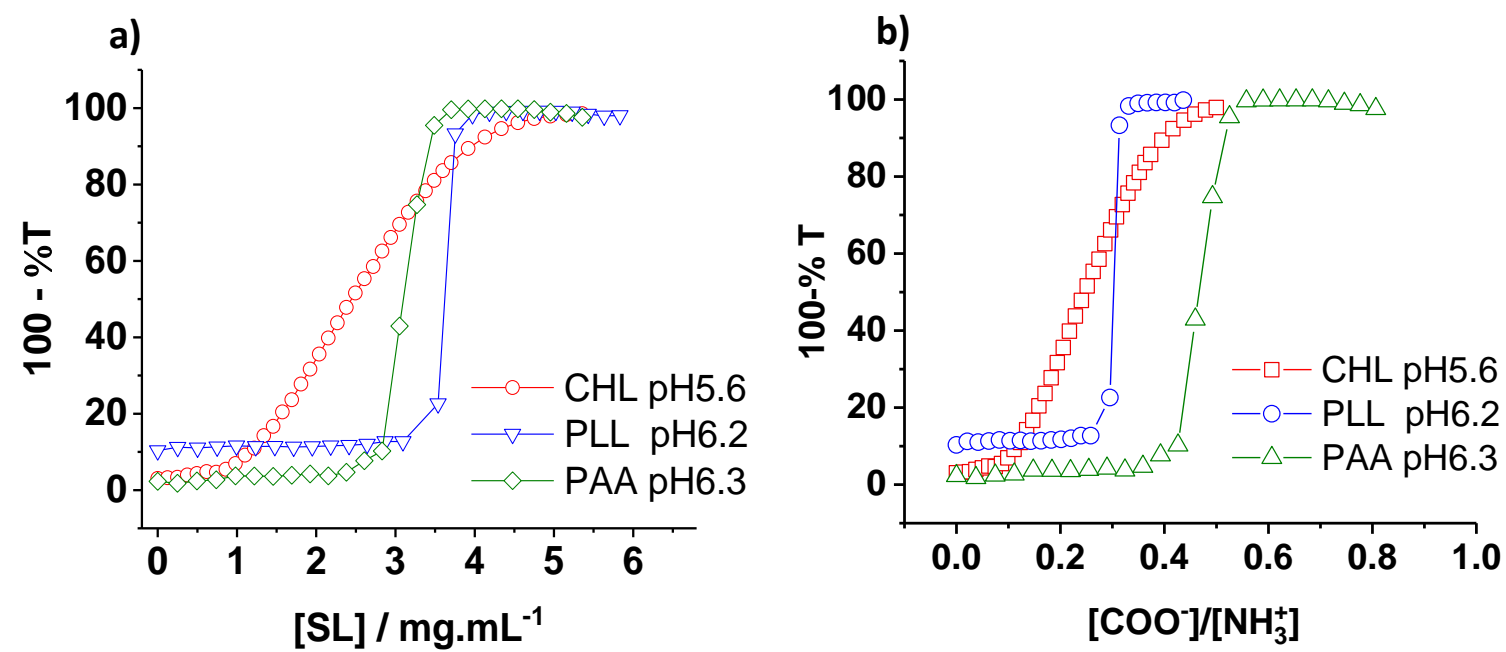

Figure S 4 - Evolution of the turbidity of PEC solutions during stepwise addition of SL solution as function of (a) SL concentration and (b) charge ratio. $[\mathrm{CHL}]=1.4 \mathrm{mg} / \mathrm{mL},[\mathrm{PLL}]=2 \mathrm{mg} / \mathrm{mL}$, and $[\mathrm{PAA}]=$ $0.75 \mathrm{mg} / \mathrm{mL}$

The difference of the critical aggregation concentration (cac) among the three systems (
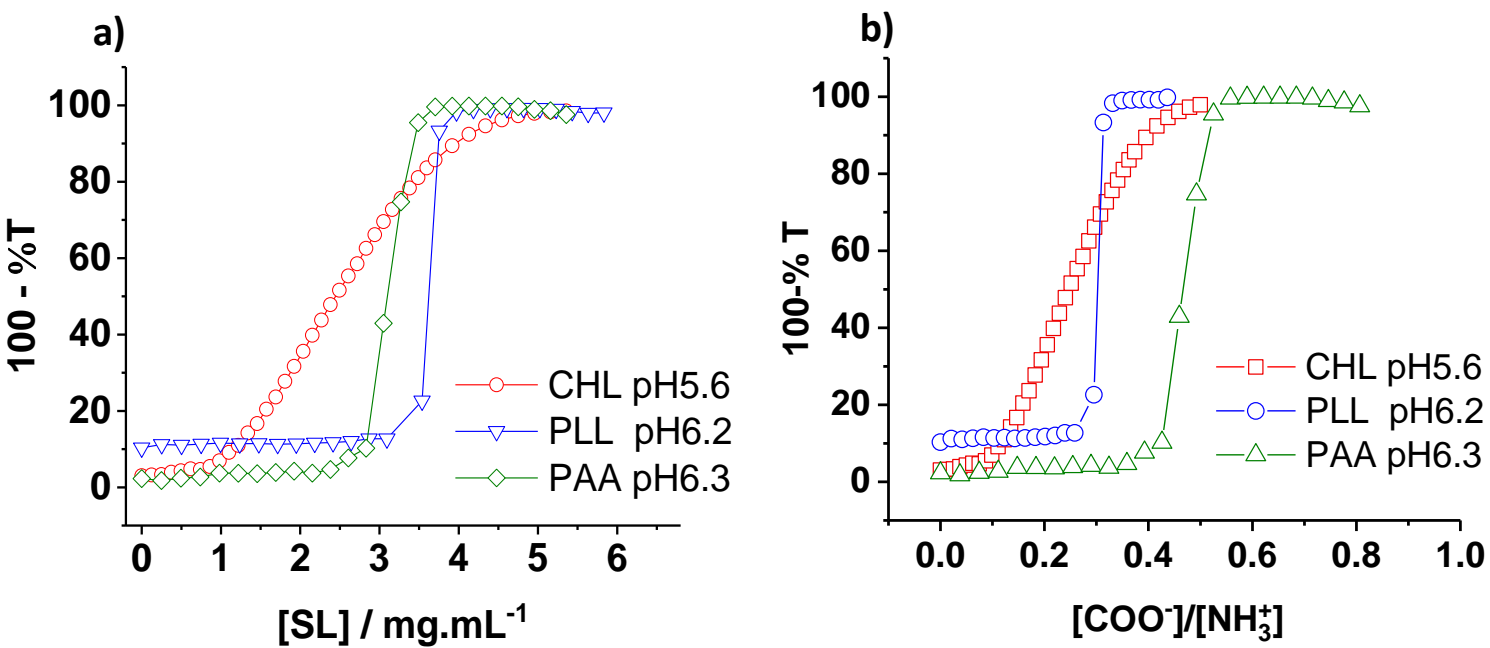

Figure $S 4 b$ ) could be related to the molecular structure of each PEC. In theory, cac is usually lower than the $\mathrm{cmc}$, however we are not able to explain the high cac compared to the $\mathrm{cmc}(0.1$ $\mathrm{mg} / \mathrm{mL}$ ) due to the complex behavior of SL. Indeed, during gradual addition of SL, free molecules can exist in solution or can even preferentially adsorb to the air-water interface to expose the intermediate aliphatic chain to the hydrophobic air phase. Furthermore, for bolaform surfactants, the relationship between the cmc and the free energy of micellization is complex and it requires taking into account the contribution of counterions. ${ }^{1}$ The analysis

\footnotetext{
${ }^{1}$ R. Zana, Critical Micellization Concentration of Surfactants in Aqueous Solution and Free Energy of Micellization. Langmuir, 1996, 12, 1208-1211.
} 
becomes even more complex due to the $\mathrm{pH}$-sensitive nature of SL. In fact, the degree of ionization, also called the apparent degree of counterions dissociation, ${ }^{2}$ will vary strongly with $\mathrm{pH}$. In addition, the counterions distribution (condensation and release during electrostatic interaction) will also be affected by the binding process of SL micelles to each PEC.

\section{Determination of the ionization degrees}

The evolution as function of the $\mathrm{pH}$ of the theoretical degrees of ionization values $\alpha$ for (SL, pKa 5.8) and $\beta$ for (CHL, pKa 6.5), (PLL, pka 10) and (PAA, pKa 9.5) were calculated as function of $\mathrm{pH}$ from the modified Henderson-Hasselbalch equation:

For SL:

(1) 13

14

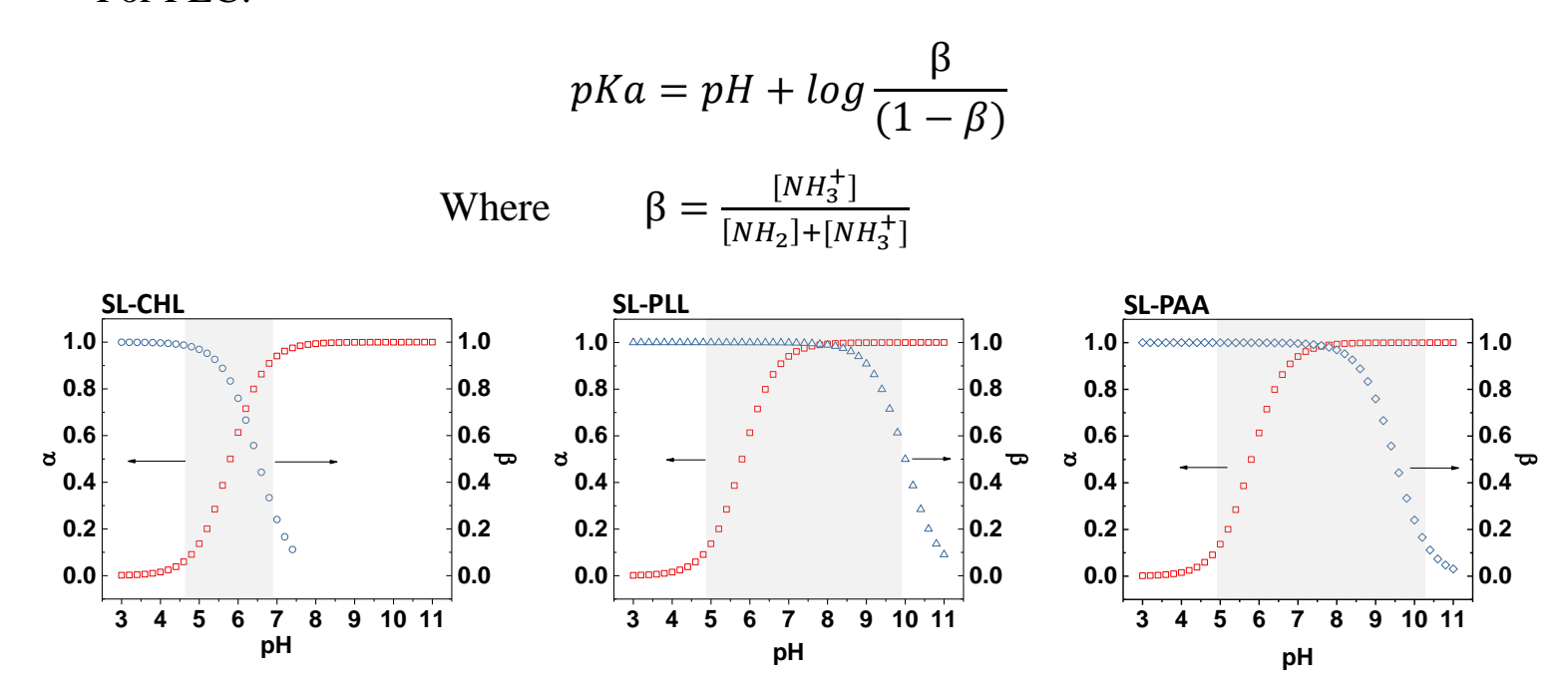

\section{For PEC:}

Where

$$
\begin{gathered}
p K a=p H+\log \frac{(1-\alpha)}{\alpha} \\
\alpha=\frac{\left[\mathrm{COO}^{-}\right]}{[\mathrm{COOH}]+\left[\mathrm{COO}^{-}\right]}
\end{gathered}
$$

Figure S 5 - Evolution of the theoretical degrees of ionization $\alpha$ and $\beta$ as function of pH for SL and polymers

\section{Determination of the charge ratio: $[\mathrm{COO}-]:\left[\mathrm{NH}_{3}{ }^{+}\right]$}

\footnotetext{
${ }^{2}$ B. L. Bales, A Definition of the Degree of Ionization of a Micelle Based on Its Aggregation Number. J. Phys. Chem. B, 2001, 105, 67986804
} 
The stoichiometric ratio for chargeable groups $(-/+)$ and defined $=[\mathrm{S}] /\left(\mathrm{n} \times[\mathrm{P}]^{3,4,5}\right.$ where $[\mathrm{S}]$ and $[\mathrm{P}]$ are the molar concentrations for the SL and for the PEC, respectively and $\mathrm{n}$ is the number of PEC monomers or binding sites. If we consider that the average molecular weight of SL, CHL, PLL and PAA are respectively 633, 4000, 5000 and 17,500 g/mol. The CHL, PLL and PAA are therefore made from average monomers of 15, 23 and 128, respectively. However, for CHL the number of binding sites is assumed to be $(15 \times 2)$ because each monomer contains two amino groups. By including the theoretical ionization degrees $(\alpha$ and $\beta)$ is therefore possible to estimate the charge ratio $\mathrm{Z}(-/+)$ or $[\mathrm{COO}-]:[\mathrm{NH} 3+]$ which will be equal to: $Z=(\alpha \times[S]) /(n \times \beta \times[P])$
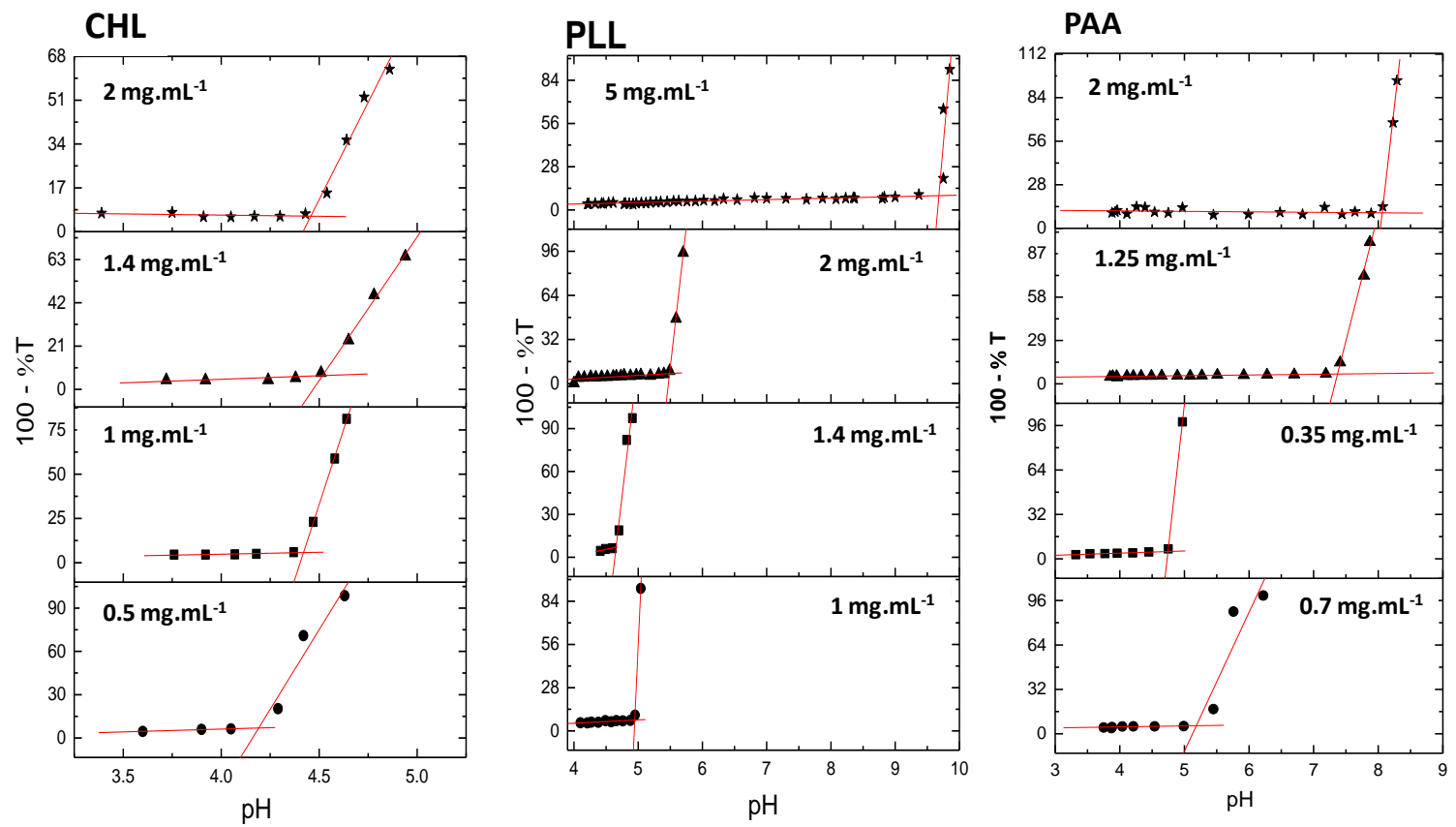

89

90

Figure S 6 - Determination of $\mathrm{pH} \phi$ as points as the intercept of the initial linear portion of the curve with the tangent to the rapidly increasing portion of the curve. $[\mathrm{SL}]=5 \mathrm{mg} / \mathrm{mL}$.

\footnotetext{
${ }^{3}$ Hervé, P., et al. "Novel core-shell structure for colloids made of neutral/polyelectrolyte diblock copolymers and oppositely charged surfactants." EPL (Europhysics Letters) 58.6 (2002): 912

${ }^{4}$ Berret, Jean-Francois, et al. "Colloidal complexes obtained from charged block copolymers and surfactants: A comparison between smallangle neutron scattering, Cryo-TEM, and simulations." The Journal of Physical Chemistry B 107.32 (2003): 8111-8118

${ }^{5}$ Berret, Jean-François, et al. "Electrostatic self-assembly of oppositely charged copolymers and surfactants: A light, neutron, and X-ray scattering study." Macromolecules 37.13 (2004): 4922-4930
} 

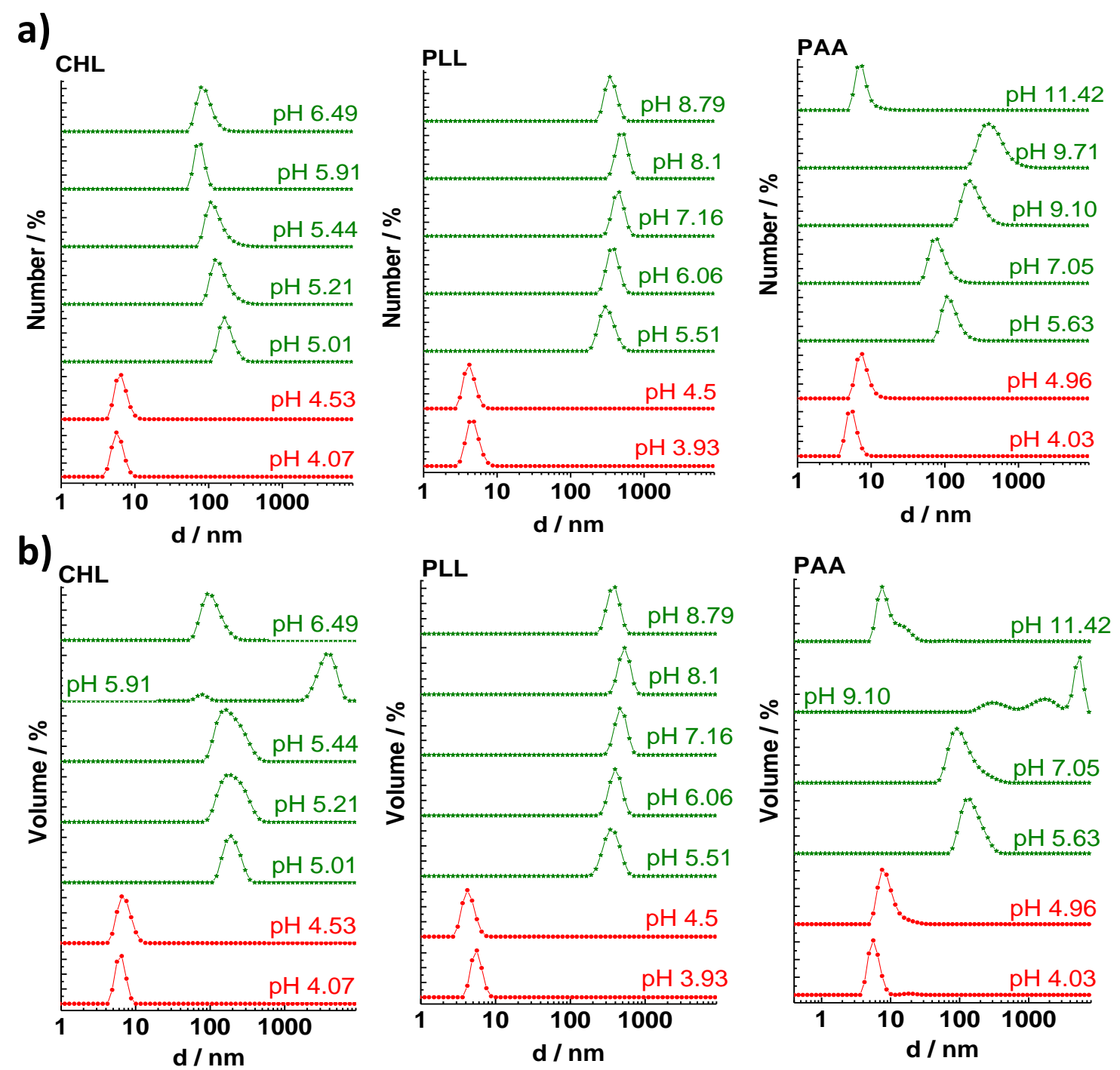

95

Figure S 7 - Evolution of the hydrodynamic diameter of SL- PEC complexes with pH, as function of the (a) relative number and (b) relative volume at $25^{\circ} \mathrm{C}$. The $\mathrm{pH}$ values are indicated above the curves. [SL]= $5 \mathrm{mg} / \mathrm{mL},[\mathrm{CHL}]=1.4 \mathrm{mg} / \mathrm{mL},[\mathrm{PLL}]=2 \mathrm{mg} / \mathrm{mL}$, and $[\mathrm{PAA}]=0.75 \mathrm{mg} / \mathrm{mL}$ 


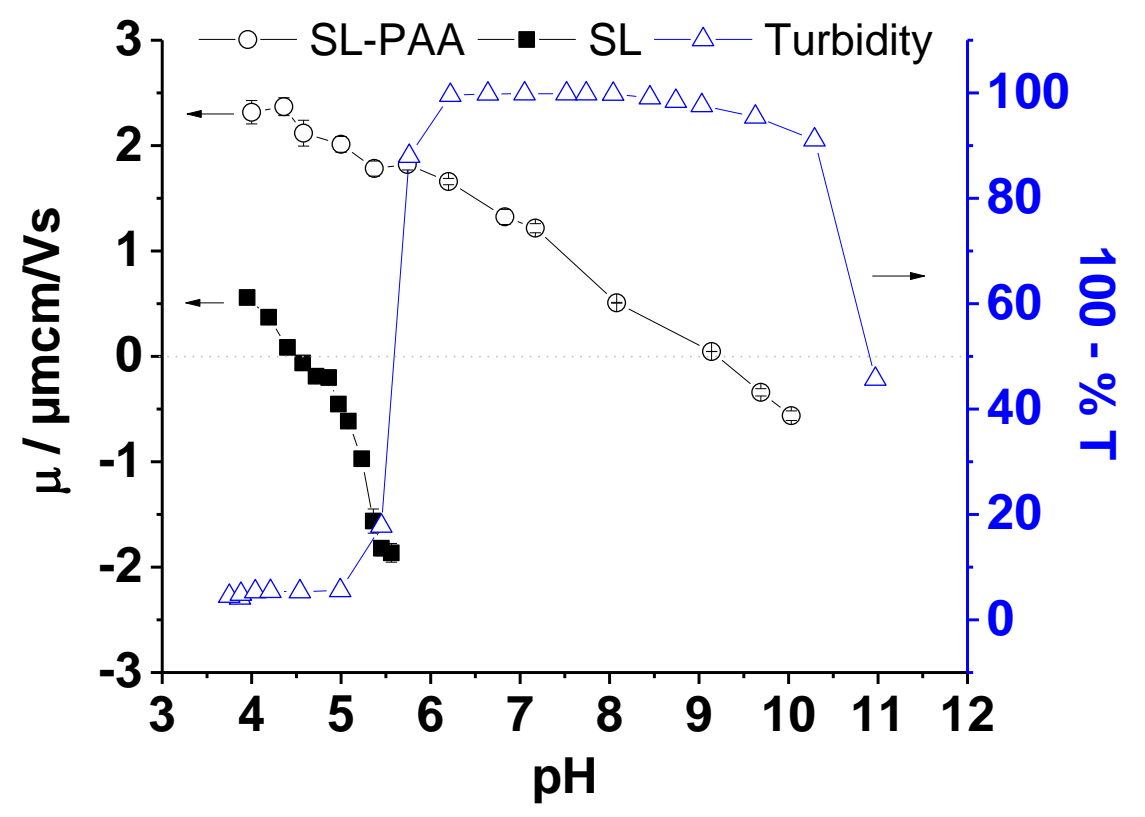

102

103

Figure S 8 - Evolution of turbidity and electrophoretic mobility as function of $\mathbf{p H}$ during complex 104 coacervation of SL-PAA ([SL] $=5 \mathrm{mg} / \mathrm{mL},[P A A]=0.75 \mathrm{mg} / \mathrm{mL})$.

105 


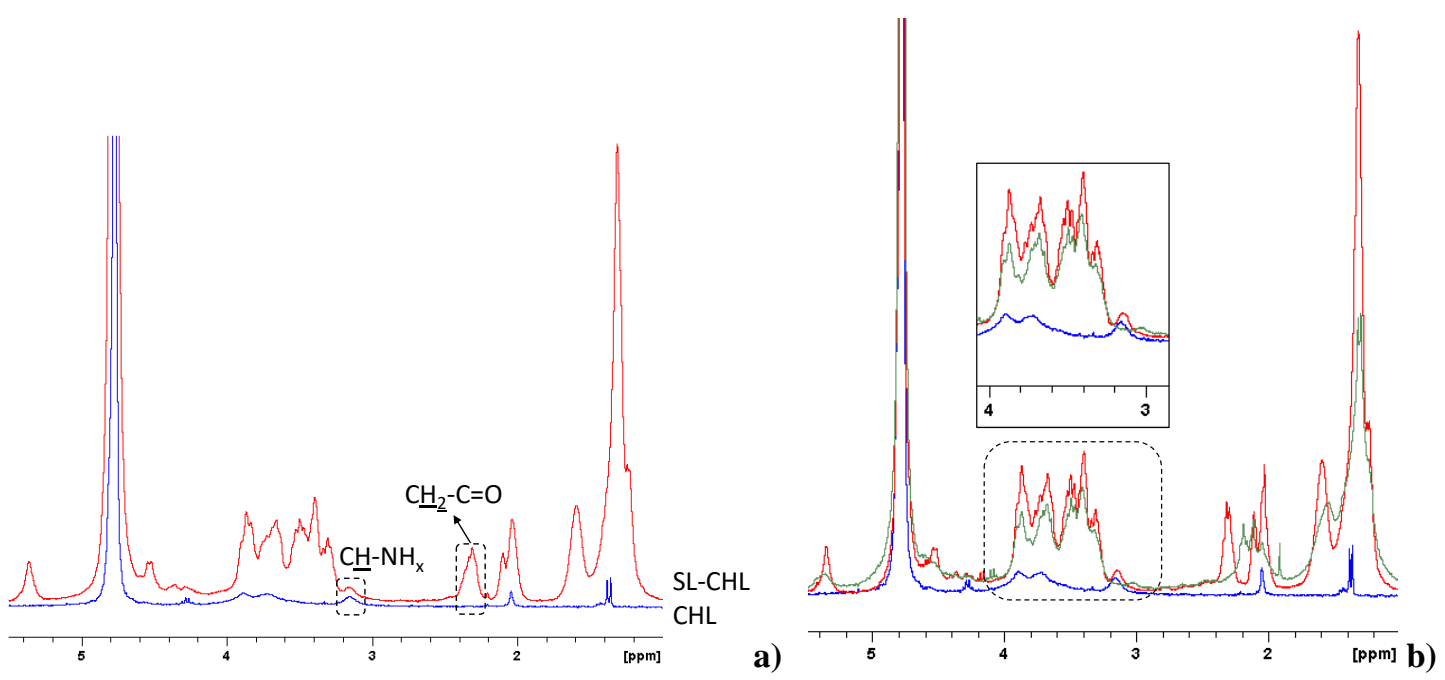

Figure $\mathrm{S} 9$ - Quantification of the a) $\mathrm{C}=\mathrm{O} / \mathrm{NH}_{\mathrm{x}}$ molar ratio in the SL-polyelectrolyte mixture in the coacervation region and b) coacervation extent. Experiments are provided for the SL-CHL system. All experiments are carried out in $\mathrm{D}_{2} \mathrm{O}$. $\mathrm{pH}$ is adjusted with $\mathrm{DCl}$ and $\mathrm{NaOD}$. The numerical data and relative discussion are provided in the main text.

a) ${ }^{1} \mathrm{H}$ NMR spectra of $\mathrm{CHL}$ solution (blue line, $\mathrm{C}=1.4 \mathrm{mg} / \mathrm{mL}, \mathrm{pH} 6.09$ ). The red signal corresponds to a SL-CHL solution initially prepared in Region 3 , on the coacervation plateau, $\left(\mathrm{C}_{\mathrm{SL}}=5 \mathrm{mg} / \mathrm{mL}, \mathrm{C}_{\mathrm{CHL}}=1.4\right.$ $\mathrm{mg} / \mathrm{mL}, \mathrm{pH} 6.12)$ and eventually centrifuged $(3000 \mathrm{rpm}, 1 \mathrm{~h})$ to recover the coacervate phase only; the coacervate is finally dispersed in $500 \mu \mathrm{L} \mathrm{D}_{2} \mathrm{O}$, intentionally set at $\mathrm{pD}<5$, out of the coacervation plateau. The $\underline{\mathrm{CH}}-\mathrm{NH}_{\mathrm{x}}$ and $\underline{\mathrm{C}}_{2}-\mathrm{C}=\mathrm{O}$ integrals are used to quantify the $\mathrm{C}=\mathrm{O} / \mathrm{NH}_{\mathrm{x}}$ molar ratio. b) ${ }^{1} \mathrm{H} \mathrm{NMR}$ spectra of SL-CHL solution $\left(\mathrm{C}_{\mathrm{SL}}=5 \mathrm{mg} / \mathrm{mL}, \mathrm{C}_{\mathrm{CHL}}=1.4 \mathrm{mg} / \mathrm{mL}\right.$ ) before (red line, $\mathrm{pH} \mathrm{4.46)}$ ) and after (green line, pH 6.12) coacervation. In blue, the signal of $\mathrm{CHL}(\mathrm{C}=1.4 \mathrm{mg} / \mathrm{mL}, \mathrm{pH} 6.09)$. The spectra are superimposed as such; no adjustment of the relative intensity is operated. The highlighted region between $4 \mathrm{ppm}$ and 3 ppm is used to measure the intensity loss before and after coacervation. 
a) SL-CHL pH 5.94

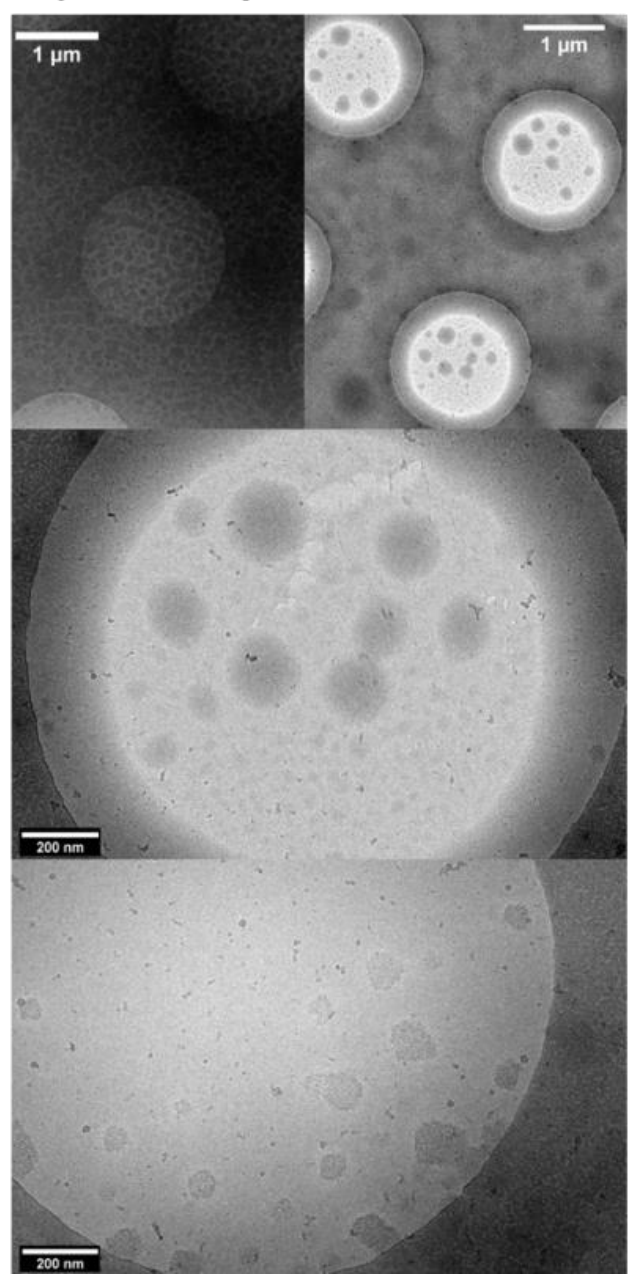

b) SL-CHL pH 6.33

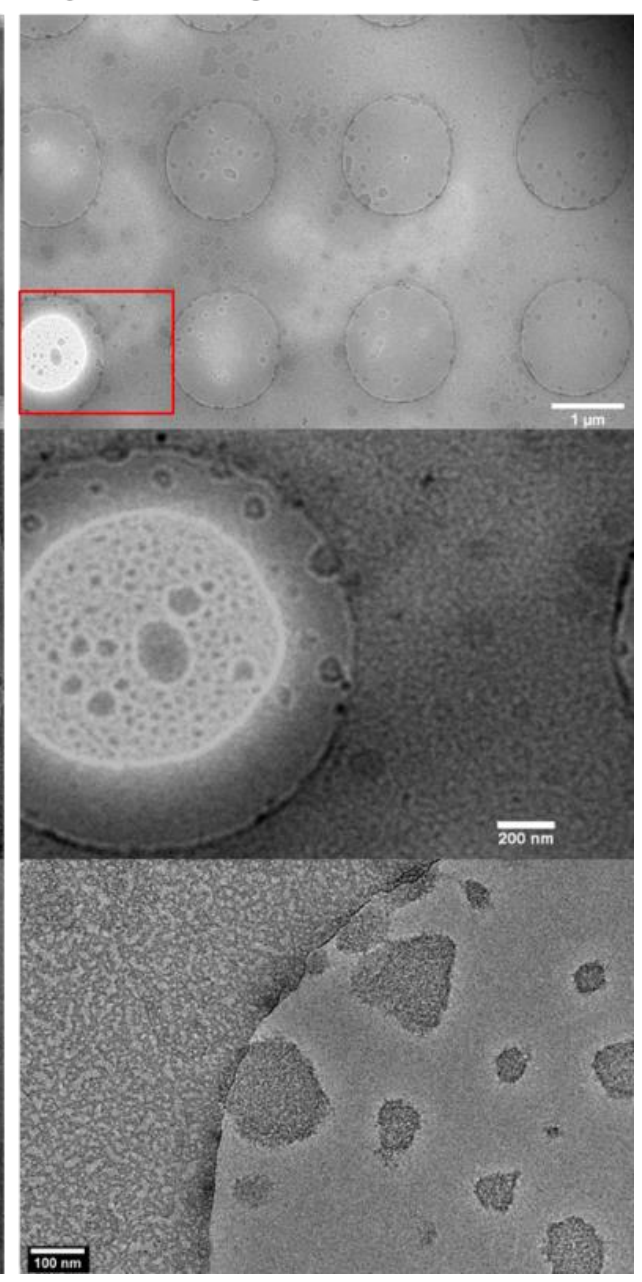

Figure S 10 - Cryo-TEM images of SL-CHL coacervates at pH 5.94 and pH 6.33 ([SL]= $5 \mathrm{mg} / \mathrm{mL}$, [CHL]= $1.4 \mathrm{mg} / \mathrm{mL})$. 


\section{SL- CHL pH 5.94}

129

130

131

132

133

134

\section{SL- CHL pH 6.33}
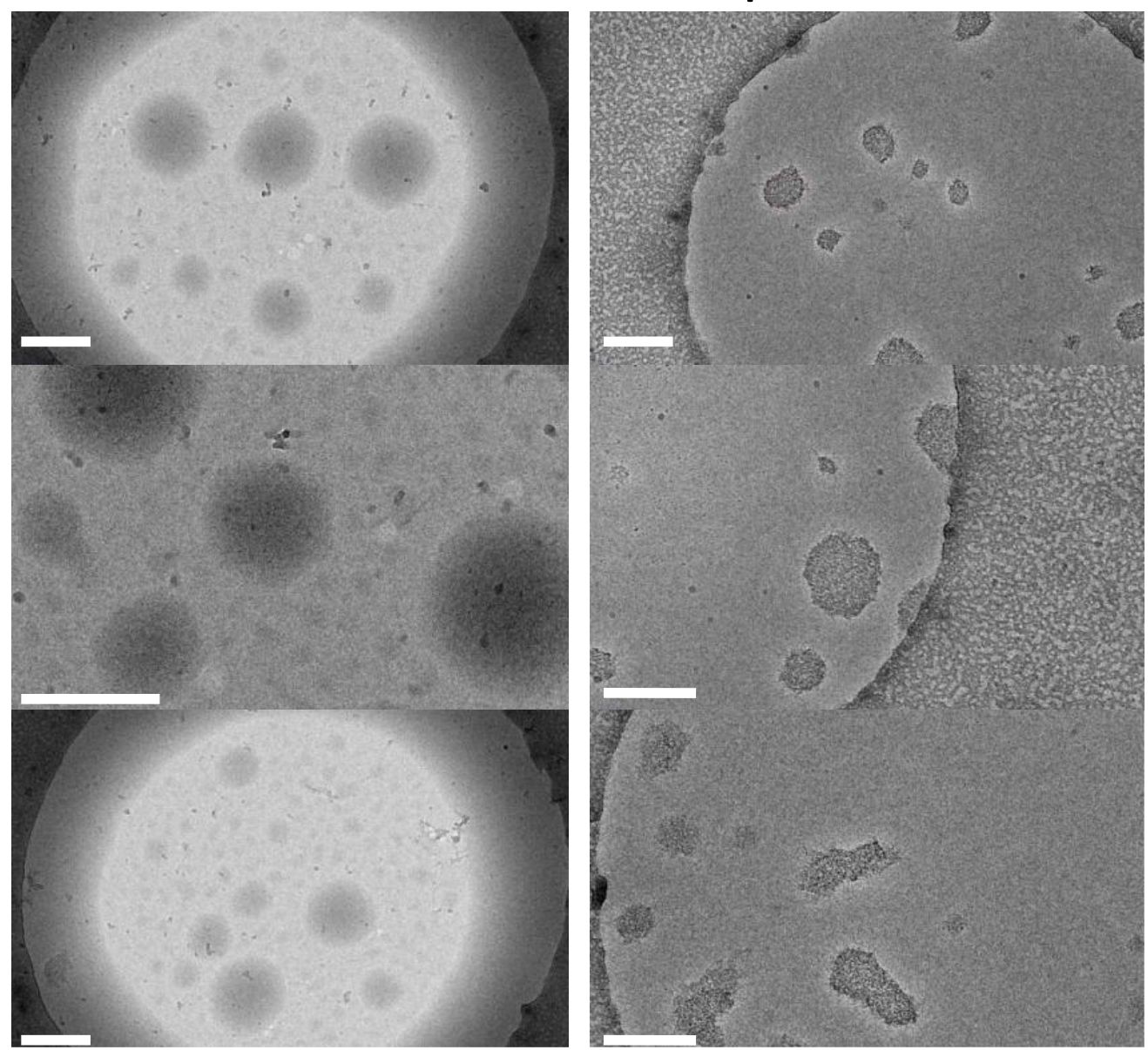

Figure S 11 - Cryo-TEM images of SL-CHL coacervates ([SL]= $5 \mathrm{mg} / \mathrm{mL}$, [CHL]= $1.4 \mathrm{mg} / \mathrm{mL}$ ). Scale bar is $200 \mathrm{~nm}$ 


\section{SL -PLL pH 5.77}

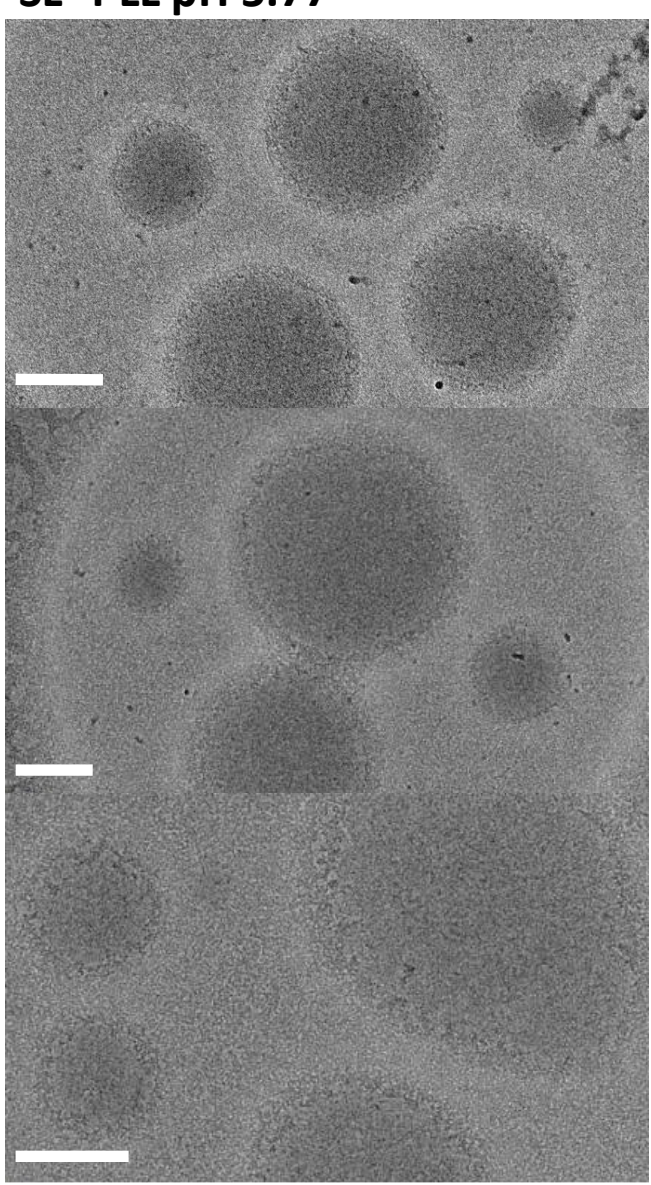

\section{SL- PLL pH 7.38}

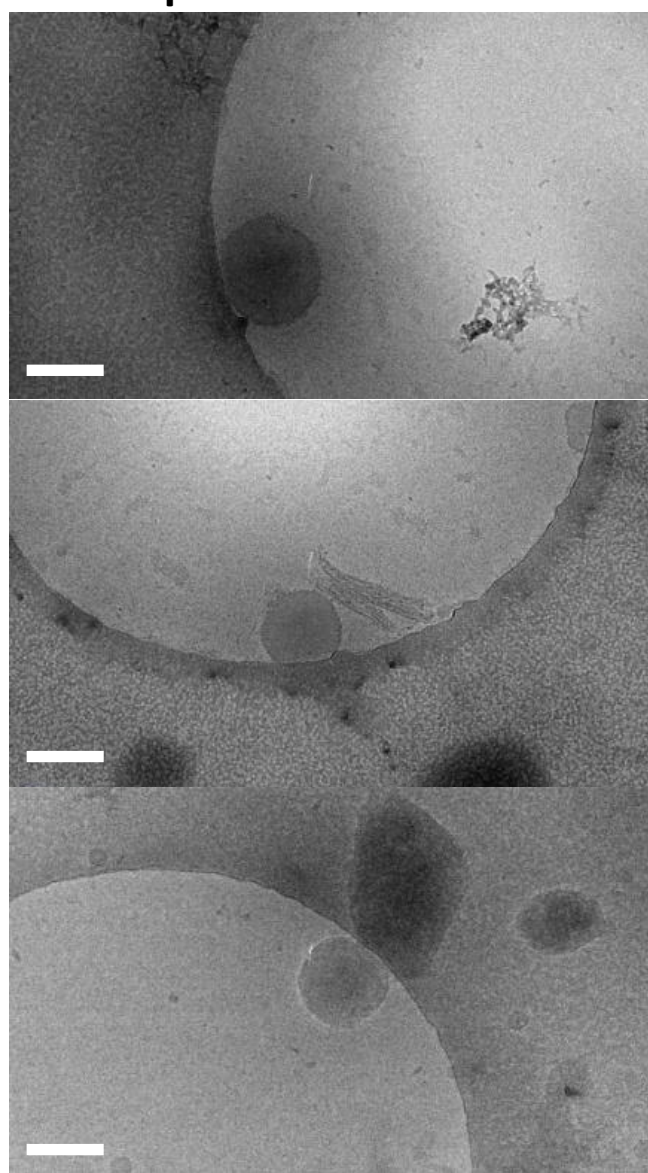

Figure $S 12$ - Cryo-TEM images of SL-PLL coacervates ([SL]= $5 \mathrm{mg} / \mathrm{mL}$, [PLL] $=2 \mathrm{mg} / \mathrm{mL}$ ). Scale bar is 138 $200 \mathrm{~nm}$ 

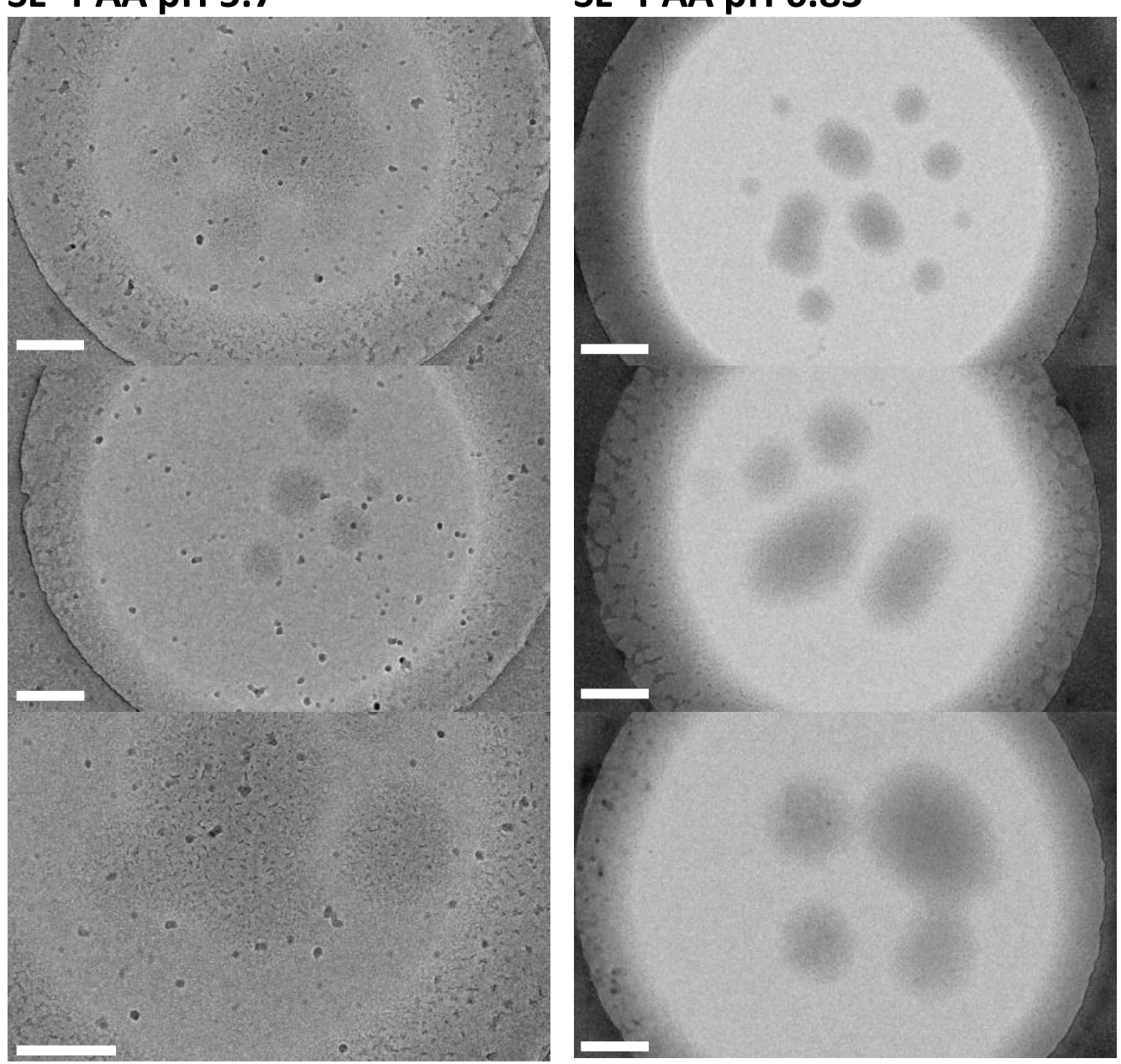

Figure S 13 - Cryo-TEM images of SL-PAA coacervates ([SL]= $5 \mathrm{mg} / \mathrm{mL}$, $[\mathrm{PAA}]=0.75 \mathrm{mg} / \mathrm{mL}$ ). Scale bar 144 is $200 \mathrm{~nm}$ 